\title{
Diet analysis of three rodent species sigmodontine in three cocoa production systems and forest in Alto Beni, Bolivia
}

\author{
Wendy Calizaya-Mena ${ }^{1 *}$, Adriana Rico-Cernohorska², Emilia García-Estigarribia ${ }^{3}$, and Esther Valenzuela-Celis ${ }^{3}$ \\ ${ }^{1}$ Carrera de Biología, Universidad Mayor de San Andrés, Campus Universitario, Calle 27 Cota Cota,10077. La Paz, Bolivia. Email: \\ wendycalizaya87@gmail.com (WC-M) \\ ${ }^{2}$ Colección Boliviana de Fauna Instituto de Ecología, Carrera de Biología, Universidad Mayor de San Andrés, Campus Universitario, \\ Calle 27 Cota Cota, 10077. La Paz, Bolivia. Email: adri_rico cer@yahoo.com; arico@fcpn.edu.bo (AR-C) \\ ${ }^{3}$ Herbario Nacional de Bolivia, Instituto de Ecología, Carrera de Biología, Universidad Mayor de San Andrés, Campus Universitario, \\ Calle 27 Cota Cota, 10077. La Paz, Bolivia. Email: emiliag9@gmail.com (EG-E), esthervalenz@gmail.com (EV-C) \\ * Corresponding author
}

Alto Beni region in Bolivia, presents a mosaic of non-extensive family crops ranging from monocultures to agroforestry systems immersed in a matrix of secondary forest, while the primary forest remains on the steeper slopes. Very little is known about the biology of the rodent species that inhabit this area. Then, the objective of this work was to determine the diet and its variation among three species of sigmodontine rodents (Akodon dayi, Euryoryomys nitidus and Oligoryzomys microtis) from 11 agroforestry systems (SAF) of cacao (four traditional monoculture / simple SAF plots, three intermediate SAF and four complex SAF) and two secondary forests. We analyzed contents of the stomach, cecum and intestine for 41 O. microtis (simple-SAF $n=31$, complex-SAF $n=4$ and forest $n=6$ ), for 11 E. nitidus (simple-SAF $n=6$, intermediate-SAF $n=2$, complex-SAF $n=3$ and forest $n=2$ ) and for 10 A. dayi (simple-SAF $n=5$ and forest $n=5$ ). We calculated trophic niche width (Levin's Index) and measured the degree of niche overlap (an estimate of competition), using Pianka's Symmetric Overlaying Index. Significant differences in the diet specialization among species across habitats, were tested using the non-parametric Kruskal-Wallis test. A total of 55 food items were obtained and grouped into nine food categories: dicotyledonous vegetative structures, monocotyledonous vegetative structures, vascular tissue, spikelet, seeds, mycorrhizae, adult arthropods, larval arthropods, and others. The study species feed primarily on plants of the family Poaceae. No significant differences were found in the diet between species or between SAF and the forest $(P>0.05)$; in addition, there was no evidence of a noticeable overlay of trophic niches by habit type. In the studied season, we found that: O. microtis and $E$. nitidus are herbivorous omnivore; "generalist" simple-monoculture-SAF and "less generalist" in complex-SAF; besides, O. microtis is "generalist" in forest and E. nitidus is "specialist" in complex-SAF and in forest; while, A. dayi is herbivore-insectivore; "generalist" in simple-monoculture-SAF and in forest. Our results indicate that there is a strong relationship between, the type of plot where they were found and food availability in each habitat.

La región de Alto Beni en Bolivia presenta un mosaico de cultivos familiares no extensivos que van desde monocultivos hasta sistemas agroforestales en una matriz de bosque secundario, donde el bosque primario se mantiene en las laderas más pronunciadas. Poco se conoce de la biología de los roderos que habitan esta zona; así, el objetivo de este trabajo fue determinar la dieta y su variación entre tres especies de roedores sigmodontinos (Akodon dayi, Euryoryzomys nitidus y Oligoryzomys microtis) procedentes de 11 sistemas agroforestales (SAF) de cacao (cuatro parcelas monocultivo tradicional/SAF-simple, tres SAF-intermedio y cuatro SAF-complejo) y dos bosques secundarios. Analizamos los contenidos en estómago, ciego e intestino de 41 O. microtis (31 en SAF-simple, cuarto en SAF-complejo y seis en bosque), once de E. nitidus (seis en SAF-simple, dos en SAF-intermedio, tres en SAF-complejo y dos en bosque) y diez de A. dayi (cinco en SAF-simple y cinco en bosque). Calculamos frecuencia, diversidad e importancia relativa de la dieta; así como, la amplitud de nicho y estimamos el grado de competencia, (índice de Sobreposición Simétrico de Pianka). Las diferencias en la dieta (entre especies y hábitats), se calculó con la prueba de Kruskal-Wallis; y se estimó el grado de especialización en la dieta. Obtuvimos 55 ítems agrupados en nueve categorías alimenticias: restos vegetales dicotiledóneas, restos vegetales monocotiledóneas, tejido vascular, espiguillas, semillas, micorrizas, artrópodos adultos, artrópodos larvas, y otros. El principal recurso alimenticio para las tres especies fueron plantas de la familia Poaceae, y la dieta no varía significativamente entre especies ni entre SAF y bosque ( $P>0.05)$. Tampoco existe marcada sobreposición de nichos tróficos por tipo de hábitats. Para la época de estudio, haIlamos que O. microtis y E. nitidus son herbívoro-omnívoros; "generalistas" en SAF-simple-monocultivo, y "menos generalista" en SAF-complejo; además O. microtis es "generalista" en bosque y E. nitidus, es "especialista" en SAF-intermedio y bosque; mientras que A. dayi es herbívoro-insectívoro; "generalista" en SAF-simple-monocultivo y en bosque. De esta manera, existe una relación entre la dieta de los roedores en relación con el tipo de parcela donde se encuentran y con la disponibilidad de alimento en cada hábitat.

Keywords: Agroforestry system; diet; diet amplitude; microhistology; rodents; Theobroma cacao.

(C) 2020 Asociación Mexicana de Mastozoología, www.mastozoologiamexicana.org

\section{Introducción}

La selección del alimento es una de las dimensiones más importantes del nicho ecológico de las especies, por lo cual la información sobre la dieta de los animales es un requisito para la mayoría de las investigaciones ecológicas (Vázquez1997; López-Cortés et al. 2007). Los roedores sigmodontinos muestran una amplia variedad de hábitos alimentarios, desde especies herbívoras hasta grados variables de omnivoría. Es posible encontrar especies frugívoras, granívoras, fungívoras y folívoras (Veloso y Bozinovic 2000; Polop et al. 2015). Sin embargo, Guabloche et al. (2002) indican que el régimen alimenticio de un roedor pequeño puede ser más variado y complejo que las categorías tróficas estándar (herbívoro o insectívoro). 
En Sudamérica se han realizado estudios sobre la dieta de algunas especies de roedores sigmodontinos (Patton et al. 2015); sin embargo, estos reportes son escasos y dispersos. Además, es común que estudios locales de dieta se extrapolen y generalicen a través de la distribución geográfica de estas especies. Por lo tanto, estos resultados no necesariamente reflejan la variación presente en la dieta de las especies, debido a la alta heterogeneidad ambiental presente a lo largo de su distribución (Veloso y Bozinovic 2000; Cervantes 2014; Polop et al. 2015).

En Bolivia, si bien la riqueza de especies de roedores es bastante amplia (Aguirre et al. 2019), y se tiene una buena información sobre sus patrones de distribución (Anderson 1997; Patton et al. 2015), no existen información detallada sobre la biología, ecología y comportamiento de estos taxones. Por lo tanto, pocos estudios han abordado la dieta de los roedores sigmodontinos, siendo el de Osco (2000) uno de los pocos estudios en donde se ha estudiado este componente en diferentes ambientes. La mayoría de los reportes son registros ocasionales de los contenidos estomacales de roedores colectados en diferentes regiones (Mercado 1991; Anderson 1997) o información generada en otros países (Vázquez et al. 2000; Suarez y Bonaventura 2001; Silva 2005; Solari 2007).

Las variaciones de la dieta pueden deberse a cambios periódicos en los factores abióticos que generan cambios en la disponibilidad temporal de recursos (Krebs 1985). La mayoría de las especies de roedores tienen comportamientos de forrajeo flexibles que permiten respuestas adaptativas a contingencias ambientales (Vázquez 1997; Vázquez et al. 2000). La forma en que los animales responden a las contingencias incluye modificaciones en sus tiempos de alimentación, distribución de su actividad y tácticas de alimentación (Brown 2000). Para los roedores, cuya dieta puede ser muy diversa, esto puede jugar un papel importante en la depredación/dispersión de semillas (Traveset 1998; Xiao et al. 2006); pero también en la depredación postdispersiva de semillas y hongos, lo cual es considerado uno de los factores principales en la regeneración natural (Pons y Pausas 2007; Campos et al. 2007). Por otro lado, algunas especies de roedores son consideradas como plagas agrícolas (Tzab-Hernández y Macswiney 2014; De la Cruz y Sánchez-Soto 2016), aunque la mayoría están especializadas en el consumo de otros ítems, tales como los artrópodos, lo cual es benéfico para la agricultura (Aubry et al. 2003; Monge 2007; Montero y Gonzáles 2017). Por otro lado, también existen especies de roedores que pueden transmitir enfermedades y son consideradas zoonóticas por el hombre (Spotorno et al. 2000; Dobson y Foufopoulos 2001). Por lo tanto, es importante conocer no solo la riqueza y abundancia de roedores en diferentes zonas, sino también su dieta, para generar adecuados procesos de control, en caso de ser determinadas como efectivas plagas agrícolas o de alerta de zoonótica.
En la región de Alto Beni, al norte del departamento de La Paz, Bolivia, las actividades antropogénicas se han incrementado en las últimas décadas. La producción de cacao en fincas familiares se incentivó a partir de 1977 (July-Martinez 2007; Bazoberry y Salazar 2008), fragmentando y destruyendo el hábitat natural de muchas especies de vertebrados (Killeen et al. 2005; Killeen et al. 2007). Actualmente, el área tiene una intensa actividad productiva de cacao (Theobroma cacao) orgánico, donde se encuentran diferentes sistemas productivos que van desde monocultivos tradicionales (sistemas agroforestales simples) hasta sistemas agroforestales (SAFs) mucho más complejos (intermedio, complejo o sucesional) (Peñafiel 2000; CATIE 2002; Somarriba y Trujillo 2005; Milz 2010; Blum 2011). Dependiendo del tipo de manejo, estos sistemas presentan distintas especies leñosas de árboles y arbustos para proporcionar sombra al cacao y cítricos (Citrus sp.), obtener forraje y hojarasca como cobertura y abono orgánico (mantillo) (Villegas y Astorga 2005). Entonces, los recursos alimenticios y de refugio variarán según el tipo de sistema productivo, generando que los roedores respondan con cambios en su forrajeo, variando la selección de su dieta.

Akodon dayi es una especie monotípica y endémica para Bolivia (Anderson 1997; Pardiñas et al. 2015). Es conocida como rata de pasto diurna y es de hábitos terrestres (Anderson y Tarifa 1996; Emmons y Feer 1997). Si bien es considerada una especie común, encontrándosela en tierras bajas ( $250 \mathrm{msnm}$ ) hasta elevaciones intermedias (hasta 2,450 msnm; Myers et al. 1990), no se tienen mayores datos sobre su biología (Anderson 1997; Eisenberg y Redford 1999; Pardiñas et al. 2015). Euryoryzomys nitidus es conocida como rata de arroz (Percequillo 2015). Se encuentra en el oeste de Brasil y en el este de Perú y Bolivia (50 a 1.985 msnm). En Bolivia se encuentra en la selva tropical de las tierras bajas de las estribaciones andinas (Anderson 1997; Musser et al. 1998). Para Bolivia no hay datos detallados disponibles sobre hábitat, hábitos, comportamiento o alimentación (Anderson 1997; Patton et al. 2000; Percequillo 2015). Oligoryzomys microtis es conocida como rata pigmea del arroz, y tiene una amplia distribución que va desde el noroeste de Brasil, sur del río Amazonas hasta tierras bajas de Perú y Bolivia (Musser y Carleton 2005; Weksler y Bonvicino 2015). Es una especie de hábitos terrestres, común en gran parte de su amplia distribución. Debido a su capacidad de adaptarse a ambientes con cierto grado de degradación, puede llegar a ser considerada plaga agrícola (Eisenberg y Redford 1999; Patton et al. 2000). Se la considera una especie principalmente granívora, frugívora e insectívora.

De acuerdo con la hipótesis del disturbio intermedio (Connell 1978) es posible que en nuestro sistema de estudio exista una mayor riqueza de especies de roedores en los SAFs intermedios/complejos de cacao en comparación con los SAF simples con manejo orgánico como consecuencia de los distintos regímenes de perturbación. Es de esperar también que la dieta de los roedores será más selectiva y 
con una menor amplitud de nicho trófico en los SAFs intermedios/complejos debido a una mayor competencia entre las especies, mientras que en los SAF simples, estos roedores tendrán dietas más generalistas y con mayor amplitud de nicho trófico. El objetivo de este estudio fue analizar la dieta de tres especies de roedores sigmodontinos (Akodon dayi, Euryoryzomys nitidus y Oligoryzomys microtis) que habitan en diferentes tipos de sistemas de producción de cacao (Theobroma cacao) y bosque secundario circundante y determinar sí existe variación en la misma en función del tipo de sistemas de producción de cacao y bosque en el que se encuentran.

\section{Material y Métodos}

Área de estudio. El presente estudio se realizó en la región de Alto Beni, ubicado al noreste del departamento de La Paz a una distancia de $270 \mathrm{~km}$ de la ciudad. Su extensión es de 250,000 ha $\left(15.182^{\circ} \mathrm{S}\right.$ y $\left.66.927^{\circ} \mathrm{W}\right)$, de las cuales el $50 \%$ corresponden a bosque primario con tendencia descendente (Navarro y Maldonado 2002; Navarro 2011). Su altitud oscila entre 350 y $1500 \mathrm{msnm}$ la temperatura media anual mínima es de $11^{\circ} \mathrm{C}$ y la máxima es de $35^{\circ} \mathrm{C}$ con una temperatura promedio anual de $26{ }^{\circ} \mathrm{C}$; el área presenta una humedad relativa promedio anual de 70 y 80 \% (Vega 2005; Ortiz -Gonzáles 2006). En zonas bajas (Covendo, $15.792^{\circ} \mathrm{S}$ y $66.977^{\circ} \mathrm{W}$ ) la precipitación es de hasta 2000 mm. (Somarriba y Trujillo 2005; Blum 2011). La región de Alto Beni se caracteriza por una producción agrícola familiar intensiva. Los cultivos de mayor importancia económica son: arroz (Oryza sativa), banano (Musa sp), cacao (Theobroma cacao), café (Coffea arabica), copuazu (Theobroma grandiflora), coco (Cocos nucifera) entre otros (Peñafiel 2000; Blum 2011). Las parcelas familiares se distribuyen en la zona formando una franja de cultivos comerciales destinados para la exportación (CATIE 2002; Somarriba y Trujillo 2005; Blum 2011). La zona de estudio es un valle que se extiende por ambos márgenes del río Alto Beni, a cuyos márgenes corre la carretera desde la localidad de Sapecho hacia Covendo (de este a oeste) y de Palos Blancos que presenta bosque de pie de monte que reúne elementos de flora y fauna de la parte alta (bosque montano húmedo) y de las tierras bajas y amazónicas, por lo que la riqueza de especies es muy alta en comparación con otras regiones del país.

Trabajo de campo. El presente trabajo se realizó en el marco del proyecto "Investigación sobre funciones ecosistémicas en sistemas de producción de cacao en Alto Beni". Se establecieron 15 parcelas de muestreo con la finalidad de mantener el muestreo lo más balanceado posible. Sin embargo, en dos de ellas no se colectó ningún roedor. Por lo que se trabajó en 13 parcelas, 11 con diferente tipo de producción de cacao y dos bosques intervenidos ubicadas a lo largo de la carretera entre Palos Blancos y Covendo. Las parcelas estuvieron separadas al menos $500 \mathrm{~m}$ entre ellas con la finalidad de mantener la independencia de los datos. De las 11 parcelas, cuatro fueron agroforestales simples o monocultivos (sistema con manejo orgánico, sombra temporal con cobertura de leguminosas, aplicación de compost y abono orgánico), tres agroforestales intermedios (sistema con manejo orgánico, sombra regular donde existe entre dos y diez especies vegetales asociadas: leguminosas, frutales y maderables), cuatro agroforestales complejos o sucesiones (sistema que trata de imitar un ecosistema natural de bosque, más de diez especies frutales, maderables asociadas y de regeneración natural) y dos bosques con poca intervención (bosque secundario con baja perturbación humana con sucesión natural de especies y ningún tipo de manejo; Figura 1). El muestreo fue realizado entre agosto a septiembre y noviembre a diciembre del 2015.

En cada parcela se estableció un cuadrante de $200 \mathrm{~m}^{2}$ por sitio y se estableció una grilla de diez por diez estaciones de trampeo separadas por 10 metros entre sí, en cada parcela se colocó un total de 100 estaciones de muestreo, con 100 trampas Sherman. Las trampas fueron cebadas con una mezcla de hojuelas de avena y atún embebidos en esencia de vainilla para formar una pasta (Tarifa y Yesen 2001). El muestreo se realizó durante tres noches consecutivas en cada sitio y las trampas fueron revisadas cada mañana, siendo reemplazadas por trampas limpias cuando tenían capturas (Poleo et al. 2006).

Todos los especímenes capturados fueron recolectados siguiendo las directrices de la Sociedad Americana de Mastozoología (Sikes et al. 2016). El tracto digestivo de cada

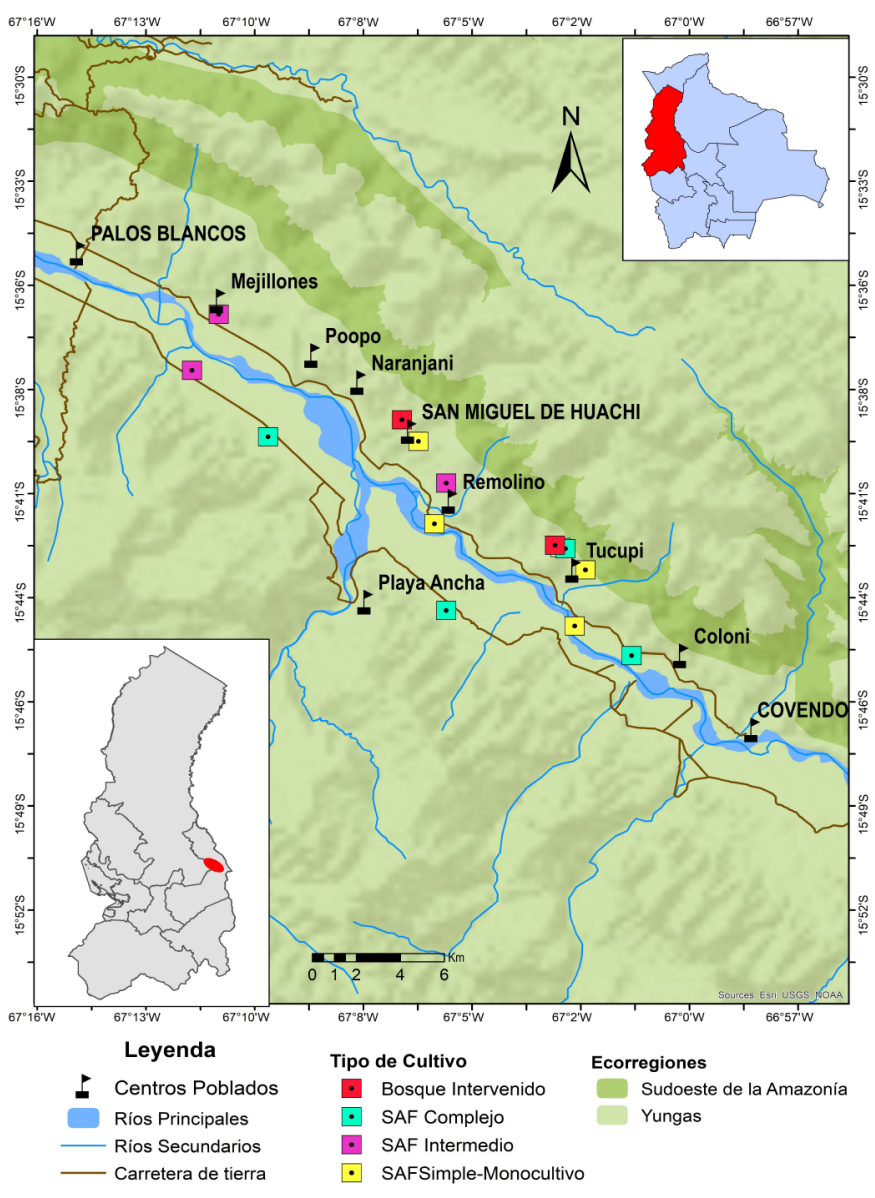

Figura 1. Mapa de la zona de estudio en el cual se indican las tres condiciones de los Sistemas Agroforestales (SAF) y bosque intervenido muestreados en este trabajo. 
individuo colectado se procesó in situ fijándolo en alcohol al $70 \%$. Los ejemplares fueron preservados en alcohol al $96 \%$ para su posterior identificación. De cada uno de los sitios de muestreo se elaboró un catálogo de referencia de especies vegetales menores a $1.5 \mathrm{~m}$ de alto presentes en el lugar, a fin de poder utilizarlo al momento de obtener los ítems alimenticios y contar con la información sobre la disponibilidad y diversidad de los recursos alimentarios de cada parcela.

Trabajodelaboratorio. Decadaespécimenseanalizaronlos contenidos del estómago, ciego e intestino (grueso-delgado; Osco 2000). Cada contenido se disgregó, homogenizó y filtró (Meserve 1981; Noblecilla y Pacheco 2012). Posteriormente fue separado en dos fracciones: partículas pequeñas $(<0.7$ $\mathrm{mm}$ ) y partículas grandes (>0.7 mm; Guabloche et al. 2002). Cada fracción fue examinada usando un estereomicroscopio a un aumento de 45X. Las partículas grandes fueron separadas y almacenadas en viales Eppendorf $(1.5 \mathrm{ml})$ para su identificación y montaje en gelatina glicerada. Las partículas pequeñas se distribuyeron homogéneamente sobre portaobjetos y se dejaron secar. Para la cuantificación de categorías alimenticias se rehidrató cada placa con una gota de alcohol al $70 \%$, se cubrió con un cubre objetos y se cuantifico usando un microscopio óptico a 40 X ó 100 $X$ aumentos (Cortés et al. 2002). Cada placa contuvo 20 campos de los cuales diez fueron evaluados (Holechek y Vavra 1981; Holechek et al.1982). Para cada uno de los órganos se realizaron tres placas y se observaron 30 campos por sector, tomando en total 90 campos por cada roedor.

Análisis de los datos. La composición de la dieta fue determinada por comparación directa con el catálogo de referencia elaborado con plantas colectadas del área de estudio (Apéndice 1). Para el análisis de los ítems alimenticios encontrados se calculó la frecuencia de presencia (Fn \%) para cada categoría en cada sector del tracto digestivo, de acuerdo con la siguiente fórmula: $\mathrm{Fn}(\%)=\left[\mathrm{N}^{\circ}\right.$ de campos donde la categoría alimenticia esta presente/ $\mathrm{N}^{\circ}$ total de campos examinados ] $\times 100$.

Para determinar la dieta de las especies de roedores en los diferentes SAFs y el bosque, los ítems alimenticios obtenidos se agruparon de acuerdo a su categoría taxonómica y tipo de recurso en restos de estructuras vegetativas dicotiledóneas y monocotiledóneas (tallos-hojas), tejido vascular (morfotipos), espiguillas (enteras y partes), semillas, micorrizas (esporocarpos-hifas), artrópodos adultos, artrópodos larvas, y otros. Para la comparación de la dieta a nivel de especies y entre tipos de hábitats se emplearon los promedios de la agrupación de cada categoría taxonómica y tipo de recurso. Para este análisis se utilizó una prueba no paramétrica de Kruskal-Wallis en el programa SPSS Statistics vers.23 (IBM Corporation. Somer, NewYork).

Para describir la diversidad en la dieta de cada especie de roedor se utilizaron datos de frecuencia de cada ítem al interior de cada categoría. Las curvas de rango-abundancia se elaboraron examinando la abundancia de dichos ítems por tipo de hábitat. Se estimó la importancia Orden/familia de los ítems vegetales o de artrópodos consumidos por las tres especies de roedores en los cuatro hábitats. Para ello se utilizaron datos de frecuencia de ocurrencia absoluta de cada ítem y se aplicó el índice de valor de importancia de los recursos (IVIR $=\Sigma(P X i)$, modificado por Amaya 1991). Este índice ha sido utilizado en análisis de la dieta de mamíferos menores (Maguiña 2011). Donde PXi es la frecuencia de uso del recurso $i$ por parte de la especie $X$ de roedor (frecuencia total de recursos utilizados por la especie $X$ de roedor). El recurso para este estudio se refiere al ítem en particular.

Para determinar el nivel de variación de uso del recurso (ítem en particular) en la dieta de cada especie, se calculó la amplitud de nicho con el Índice de Levins (Levins 1968): $B=$ $1 / \Sigma$ Pi2. Donde $B$ es la medida de amplitud de nicho trófico y $P i$ es la proporción del recurso encontrado en cada unidad de evaluación.

Posteriormente, se aplicó la ecuación de Levins estandarizada, donde valores cercanos a 0 indican que la especie es más especialista, y valores cercanos a 1 indican que es una especie generalista: $B a=(B-1) /(n-1)$; donde $B a$ es la amplitud de nicho estandarizada, $B$ es la medida de amplitud de nicho y $n$ es el número de categorías alimentarias (ítems) consumidos por la especie.

El grado de competencia por el uso de recursos alimenticios (ítems) se calculó con el índice de sobreposición simétrico de Pianka (Pianka 1973) y el índice modificado de Morisita (Horn 1966). El Índice de Sobreposición se considera alto para valores sobre 0.75 y bajo para menores a 0.50 (Noblecilla-Huiman 2008).

Se calculó la media general de cada categoría consumida (la mayor frecuencia de una categoría consumida, Dytham 2001). Posteriormente se determinó el coeficiente de variación (CV) a partir de las medias y desviaciones estándar de cada categoría. El grado de especialización en la dieta se determinó utilizando el coeficiente de variación (CV) para cada categoría alimenticia de cada especie con nueve o más individuos. En este análisis no se tomó en cuenta la condición del hábitat. Se consideraron valores de $\mathrm{CV}$ menores a $30 \%$ como indicativos de alguna preferencia para las categorías alimenticias (Solari 2007; Noblecilla-Huiman 2008; Cervantes 2014).

\section{Resultados}

En todas las parcelas de trabajo se capturaron 13 especies de roedores, de las cuales solamente Oligoryzomys microtis, Euryoryzomys nitidus y Akodon dayi contaron con el suficiente número de individuos para poder hacer el estudio. Del resto de las especies (Microryzomys minutus, Hylaeamys perenensis, Hylaeamys megacephalus, Neacomys vargasllosai, Oecomys bicolor y Rhipidomys nitela, Proechimys brevicauda y Proechimys sp.) se colectaron menos de cinco especímenes por especie. Se analizó la dieta de 64 roedores: 41 de 0 . microtis ( $n=31$ en SAF simple, $n=4$ en SAF complejo y $n=6$ en bosque), 13 de E. nitidus ( $n=6$ en SAF simple, $n=2$ en SAF intermedio, $n=3$ en SAF complejo y $n=2$ en bosque) y 10 de A. dayi ( $n=5$ en SAF simple y $n=5$ en bosque), registrándose un total de 55 ítems alimenticios (Apéndice 2). 
Oligoryzomys microtis. Esta especie consumió principalmente ítems de la categoría restos vegetales en todos los sitios donde fue colectada (Tabla 1). En SAF simple se encontró un total de 35 ítems y una baja pendiente en su gráfica de rango-abundancia indicando una mayor equitatividad de los ítems alimenticios (Figura 2). La categoría de restos vegetales monocotiledóneas fue la más abundante (33.62 \%), seguido de micorrizas (23.29\%) y restos vegetales dicotiledóneas (21.25\%; Tabla 1). Dentro de la categoría micorrizas el ítem más dominante fueron las hifas intraradicales de Glomus macrocarpum (6.70\%), respecto a los restos vegetales, la hoja de la morfoespecie Bomarea sp. (5.27 \%) fue la más consumida, seguida de los tallos de Paspalum conjugatum (4.16\%) y Paspalum paniculatum (4.44 \%) (plantas monocotiledóneas). En menor porcentaje se registraron tallos de Hydrocotyle sp. (4.73\%) y de Acalypha stricta (3.08\%; plantas dicotiledóneas). Entre los artrópodos adultos, el orden Hymenoptera (2.08 \%) fue el más consumido, seguido de la familia Formicidae (1.18\%) y el orden Hemiptera en menor frecuencia (0.97\%; Figura 2). En SAF complejo, los 12 ítems consumidos presentan una curva de rango-abundancia con mayor pendiente y menor equitatividad que para SAF simple. La categoría más abundante fue restos vegetales monocotiledóneas $(81.11 \%)$, seguido de la categoría otros $(14.45 \%)$ y restos vegetales dicotiledóneas (4.44\%). En esta última, los tallos de Axonopus compressus (21.11\%), Imperata sp. (18.61\%) y Oplismenus hirteIlus (16.67 \%; monocotiledóneas) fueron los más frecuentes (Figura 2). En las parcelas de bosque también se registraron 12 ítems y una curva similar a SAF complejo (Figura 2). Se registró un mayor porcentaje de restos vegetales dicotiledóneas $(28.33 \%)$, seguido de restos vegetales monocotiledóneas (27.78\%), espiguillas (22.78 \%) y la categoría otros (21.11\%), donde los tallos de Paspalum paniculatum (20.00 \%; monocotiledónea) e Ipomoea sp. (11.67 \%; dicotiledónea) fueron los ítems más frecuentes. O. microtis fue la única especie que presentó restos de alga Chlorophyta (4.44\%) en su dieta. El análisis de la amplitud de nicho indica que $O$. microtis tiene una dieta amplia o generalista en parcelas SAF simple-monocultivo, catalogándolo como omnívoro. En SAF complejo es herbívoro, ya que consume principalmente restos vegetales presentando una dieta menos amplia por ser más especialista. En bosque, es omnívora, de dieta amplia y más generalista, que en SAF complejo (Tabla 2).

Euryoryzomys nitidus. Esta especie presentó una dieta más omnívora, donde las categorias mas importantes variaron según el tipo de sistema de producción (Tabla 1). EI SAF simple presentó mayor número de ítems alimenticios en su dieta, con 20 en total y una curva de rango-abundancia con una pendiente moderada (Figura 3). La categoría artrópodos adultos (26.66\%) fue la más abundante, seguida de la categoría otros (24.07\%) y espiguillas (22.41\%). Las categorías restos vegetales monocotiledóneas (10.75\%), dicotiledóneas (7.78 \%), artrópodos larvas (7.22 \%) y tejido vascular (morfotipos; $1.11 \%$ ) fueron menos abundantes. Para los artrópodos adultos, el ítem Coleóptera (22.96 \%) fue el más dominante, aunque también se registraron larvas de Hymenoptera (7.22\%) en este sistema de producción. En el SAF intermedio, se registraron 12 ítems alimenticios distribuidos de manera más equitativa. La categoría restos vegetales monocotiledóneas fue la más abundante $(32.23 \%)$ seguida de la categoría otros (31.67\%) y restos vegetales dicotiledóneas (18.34\%), donde los ítems tallos de Cyperus chalaranthus (15.56 \%; monocotiledónea) e Iresine diffusa (10.56 \%; dicotiledónea) y hojas de Anthurium sp. (8.89\%; monocotiledónea) fueron los más frecuentes. No se reportó consumo de artrópodos ni micorrizas en este tipo de sistema, pero sí ítems de las categorías espiguilla: Paspalum paniculatum (12.20\%; monocotiledónea) y semillas: Polygonum sp. (5.56 \%; dicotiledónea). El SAF complejo también presentó 12 ítems alimenticos presentando una curva de rango abundancia

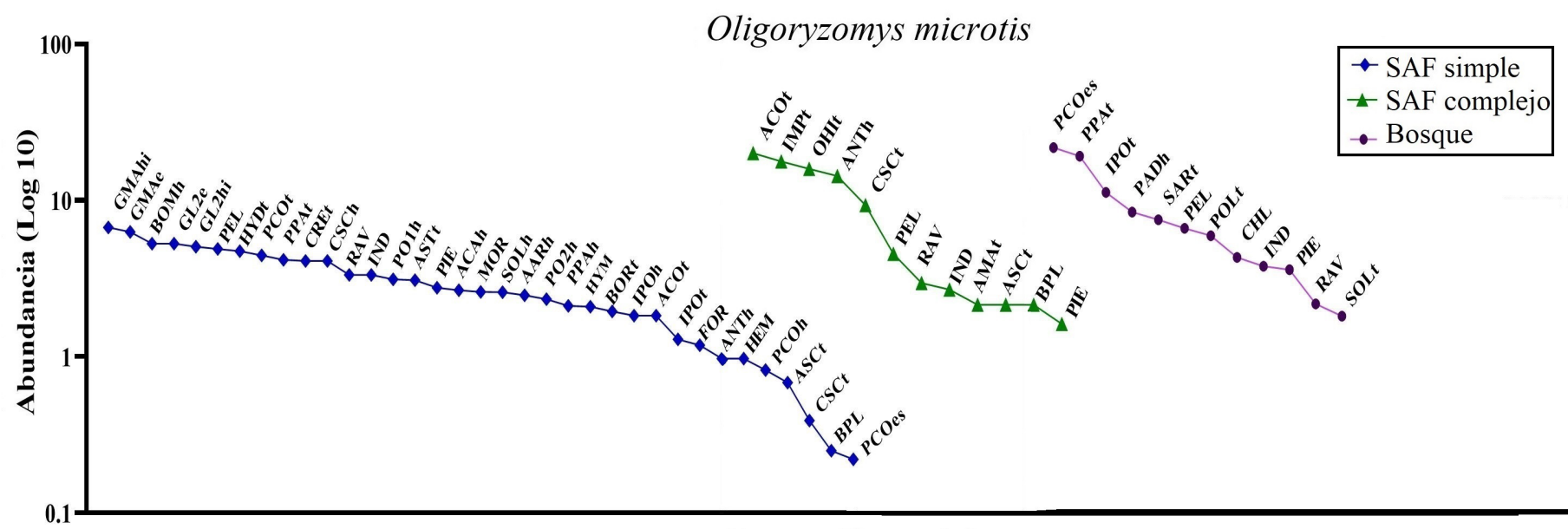

\section{Items alimenticios}

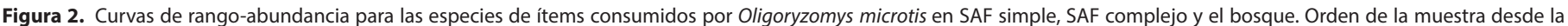

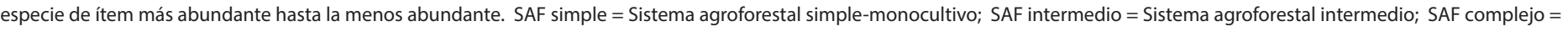

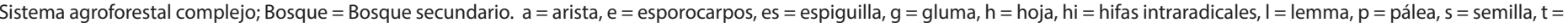

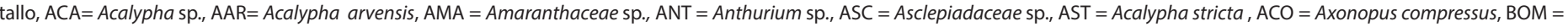

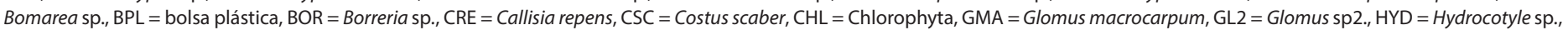

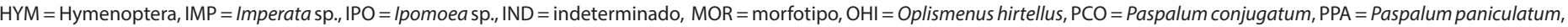

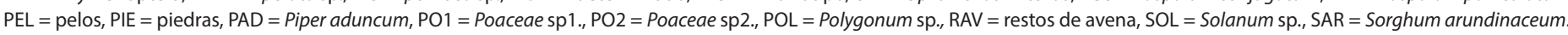




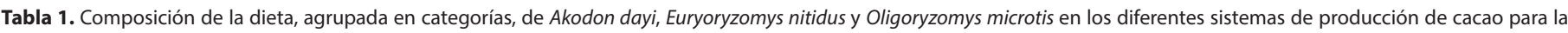
época de transición húmeda-seca. Se muestra la ocurrencia de individuos y sus porcentajes (entre paréntesis) en cada sitio de muestreo.

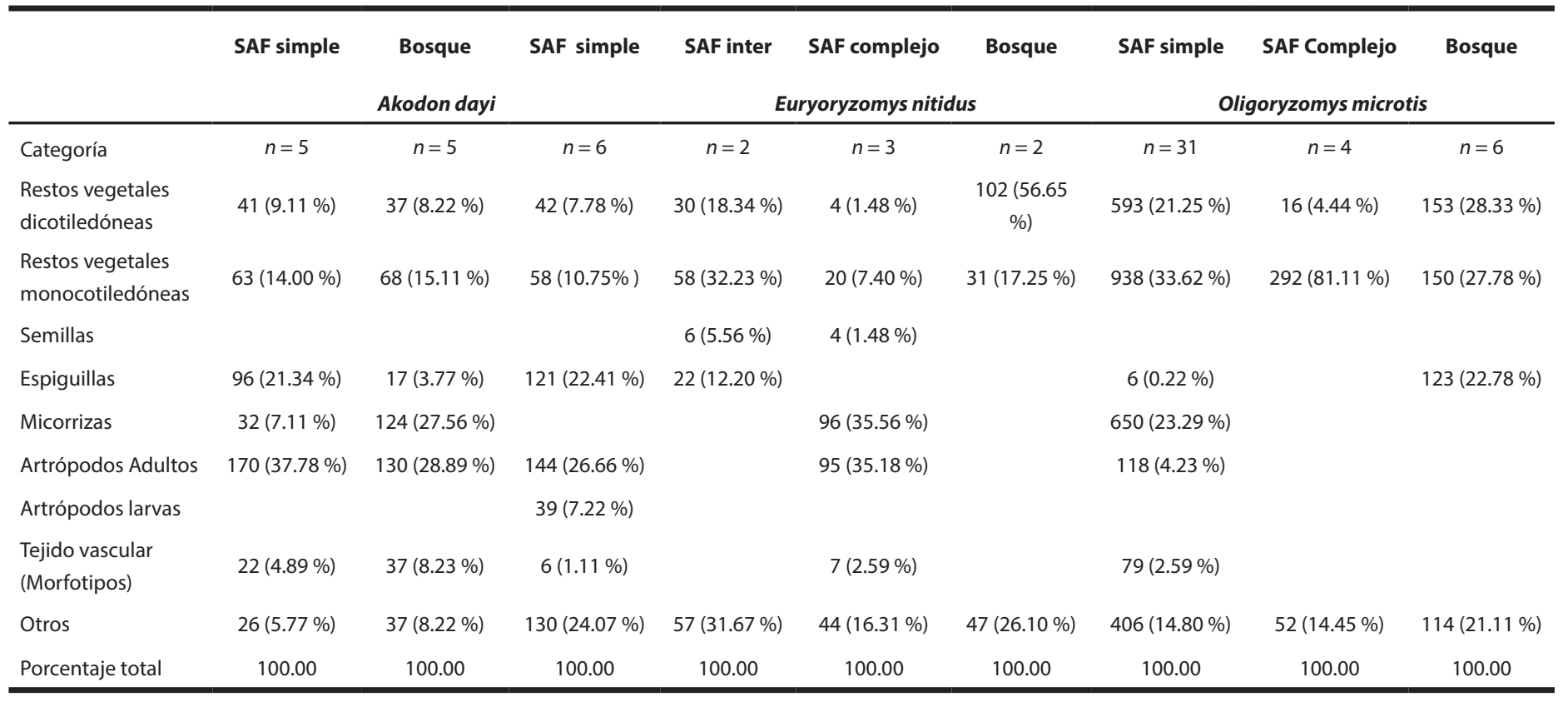

SAF simple = Sistema agroforestal simple-monocultivo; SAF inter = Sistema agroforestal intermedio; SAF complejo = Sistema agroforestal complejo; Bosque = Bosque secundario.

con mayor pendiente que en los dos anteriores sistemas (Figura 3). Las categorías con mayor consumo fueron micorrizas (35.56 \%), artrópodos adultos (35.18\%) y otros (16.31\%). Las categorías menos consumidas fueron restos vegetales monocotiledóneas (7.40 \%), tejido vascular (morfotipos; $2.59 \%$ ), restos vegetales dicotiledóneas $(1.48 \%)$ y semillas (1.48\%). En la categoría de micorrizas se encontraron en mayor frecuencia restos de esporocarpos de Glomus clavisporum (35.56\%); en la de artrópodos adultos, los ítems Coleóptera (21.48 \%) e Hymenoptera (13.70\%) fueron los más frecuentes; mientras que, en restos vegetales, los restos de hoja de Anthurium sp. (4.44 \%) y de tallo de Cyperus chalaranthus (2.96 \%; monocotiledóneas) fueron las más frecuentes. El bosque presentó el menor número de ítems, con solo 10 y una pendiente pronunciada
(Figura 3). En el bosque esta especie mostró una preferencia por restos vegetales dicotiledóneas $(56.65 \%$ ) y la categoría otros $(26.10 \%)$, y en menor preferencia a los restos vegetales monocotiledóneas (17.25\%); el ítem hoja de Desmodium sp. (33.33\%; dicotiledónea) fue el más frecuente, pero también se hallaron restos de tallo de Oplismenus hirtellus (13.89\%) (monocotiledónea), el tallo Acalypha stricta (12.22 \%) y la hoja de Acalypha arvensis (8.89\%) (dicotiledóneas). En estas parcelas tampoco se reportaron restos de artrópodos y micorrizas. El análisis de amplitud del nicho indica que E. nitidus en parcelas del SAF simple-monocultivo es un consumidor generalista y de dieta amplia, catalogándolo como omnívoro. En el SAF complejo es generalista y de dieta menos amplia comparada con SAF simple; mientras en parcelas SAF intermedio y bosque es herbívoro y

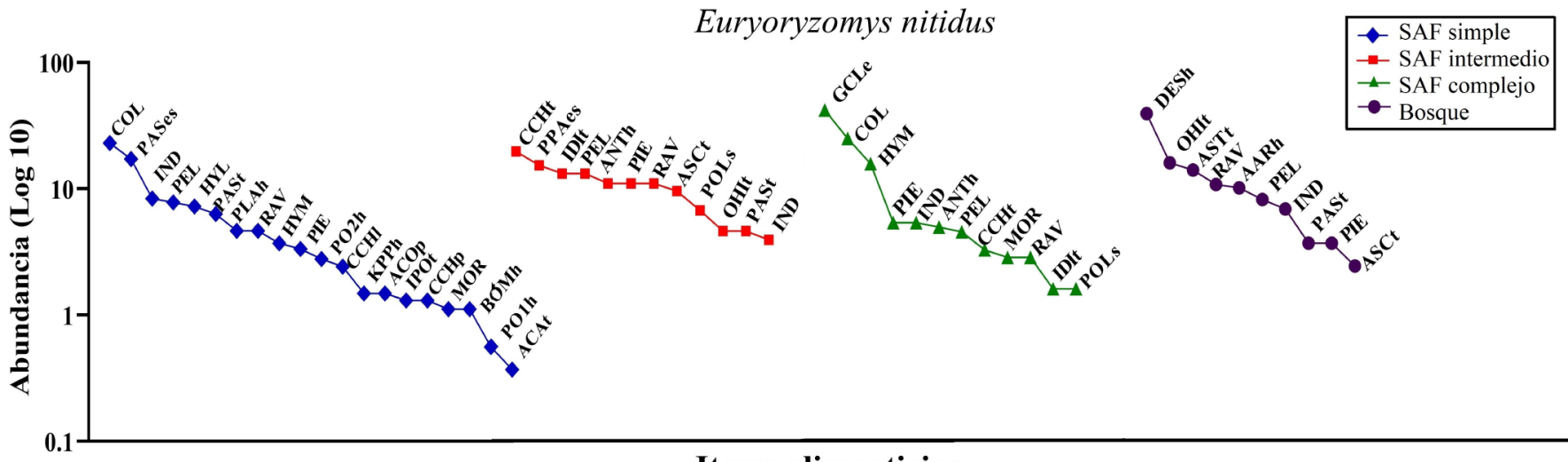

\section{Items alimenticios}

Figura 3. Curvas rango-abundancia para las especies de ítems consumidos por Euryoryzomys nitidus en SAF simple, SAF intermedio, SAF complejo y el bosque. Orden de la muestra

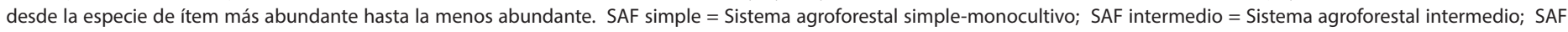

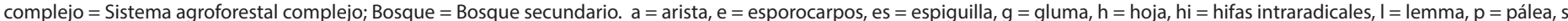

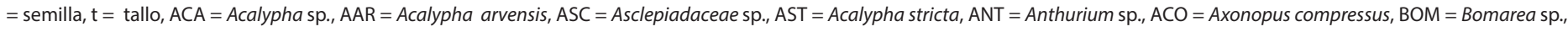

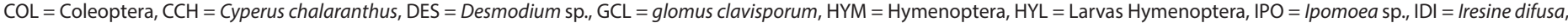

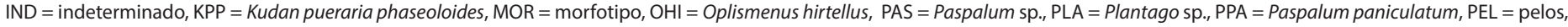
$\mathrm{PIE}=$ piedras, $\mathrm{PO} 1=$ Poaceae sp1., $\mathrm{PO} 2=$ Poaceae sp2., $\mathrm{POL}=$ Polygonum sp., $\mathrm{RAV}=$ restos de avena. 
Tabla 2. Valores del índice de Levins y Levins estandarizado para la dieta de tres especies de roedores sigmodontinos en diferentes sistemas de producción de cacao (Theobroma cacao), en Alto Beni (La Paz-Bolivia) durante el periodo de transición húmeda-seca.

\begin{tabular}{llrr}
\hline Especie & \multicolumn{1}{c}{ Sitio } & $\begin{array}{c}\text { Índice de } \\
\text { Levins } \boldsymbol{B}\end{array}$ & $\begin{array}{c}\text { Levins } \\
\text { estandarizado } B \boldsymbol{A}\end{array}$ \\
\hline \multirow{3}{*}{ Oligoryzomys microtis } & SAF simple & 26.54 & 0.65 \\
& SAF complejo & 6.91 & 0.54 \\
& Bosque & 9.26 & 0.64 \\
& SAF simple & 10.47 & 0.73 \\
Euryoryzomys nitidus & SAF inter & 8.06 & 0.32 \\
& SAF complejo & 5.80 & 0.53 \\
& Bosque & 3.66 & 0.33 \\
Akodon dayi & SAF simple & 25.82 & 0.75 \\
& Bosque & 18.51 & 0.70 \\
\hline
\end{tabular}

SAF simple = Sistema agroforestal simple-monocultivo; SAF inter = Sistema agroforestal intermedio; SAF complejo = Sistema agroforestal complejo; Bosque $=$ Bosque secundario.

consumidor especialista, ya que consume principalmente restos vegetales (Tabla 2).

Akodon dayi. Esta especie consumió principalmente ítems de la categoría artrópodos adultos en todos los sitios donde fue colectada. Al igual que para las otras dos especies, también se encontró una mayor cantidad de ítems alimenticios en SAF simple, 28 en total (Figura 4), donde la pendiente de la curva de rango abundancia es muy poco pronunciada. La categoría más consumida fue artrópodos adultos (37.78\%); donde los ítems: restos de los órdenes Hymenoptera (13.56 \%), Coleóptera (10.67 \%) y Díptera (5.56 \%) fueron los más consumidos. La categoría espiguillas (21.34\%) también fue muy consumida, donde el ítem Paspalum paniculatum (5.11 \%) y Paspalum conjugatum (4.89\%) fueron las más frecuentes. Las categorías restos vegetales monocotiledóneas (14.00 \%) y dicotiledóneas $(9.11 \%)$ fueron menos consumida, donde el ítem hoja de la morfoespecie Hydrocotyle sp. (5.78\%; dicotiledónea) fue la más frecuente. Para las parcelas de bosques se reportó un total de 20 ítems diferentes en la dieta de $A$. dayi y una pendiente poco pronunciada en la curva de rango-abundancia (Figura 3). Las categorías más consumidas fueron artrópodos adultos (28.89\%), micorrizas $(27.56 \%)$ y restos vegetales monocotiledóneas (15.11\%) y dicotiledóneas (8.22 \%; Figura 4). Con relación a los ítems de artrópodos adultos, los órdenes más frecuentes fueron Hymenoptera, pero en específico la familia Formicidae (12.22 \%), otros Hymenoptera (7.33 \%) y Díptera (6.22 \%). No se registraron restos de coleópteros. Los restos de esporocarpos de Glomus fasciculatum (10.22 \%) y Glomus spp. (7.11\%), se reportan por primera vez en la dieta de esta especie. Akodon dayi se cataloga como generalista, de dieta amplia, según el análisis de amplitud de nicho; tanto en parcelas SAF simple-monocultivo, como en bosque intervenido (Tabla 2).

De acuerdo con los valores del IVIR las tres especies de roedores tienen como principal recurso en su dieta plantas de la familia Poaceae con 0.772 de IVIR en SAF simplemonocultivo; 0.233 de IVIR en SAF intermedio; 0.564 IVIR en SAF complejo y 0.754 de IVIR en bosque (Apéndice 3 ). En el SAF simple el orden de hongos Glomerales es la más importante para la especie O. microtis, el orden Coleóptera para E. nitidus y el orden Hymenoptera para A. dayi. En el bosque, el orden Glomerales es la más importante para A. dayi, mientras que para $E$. nitidus lo son las familias Papilionaceae $y$ Euphorbiaceae. Por otro lado, los resultados del IVIR en SAF intermedio (tomando valores solo de E. nitidus) mostraron que el recurso principal es la familia Poaceae con 0.21 de IVIR. En el SAF complejo (sumando los valores de dos especies) mostraron como principal recurso a la familia Poaceae con 0.56 de IVIR. Sin embargo, el uso de los recursos varía entre las tres especies y entre los sitios estudiados (Tabla 3, Apéndice 3).

De manera general, en el SAF simple las tres especies de roedores consumen en mayor frecuencia restos vegetales. En el SAF intermedio la única especie presente, E. nitidus, tuvo restos vegetales como su categoría principal de

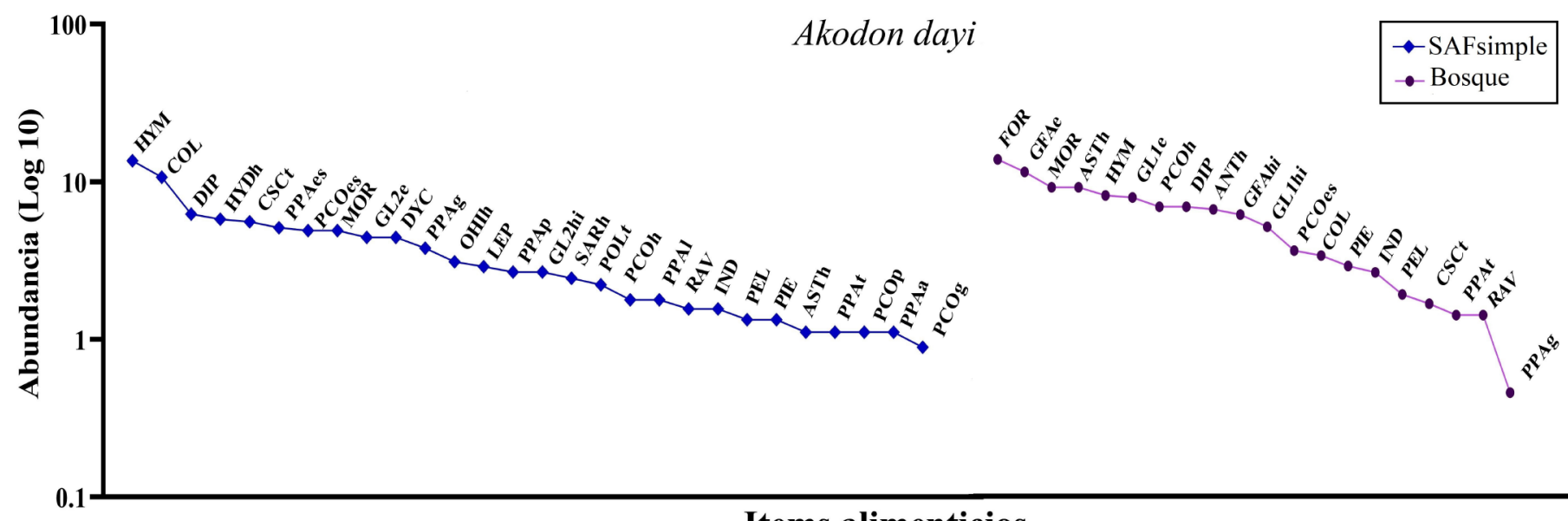

\section{Items alimenticios}

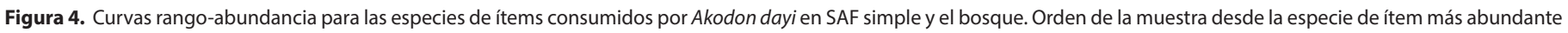

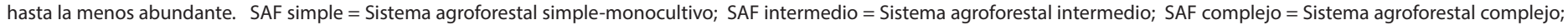

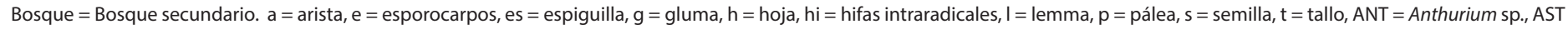

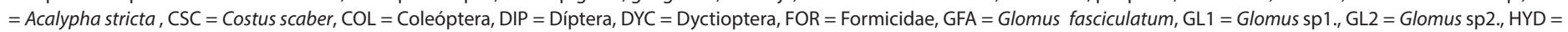

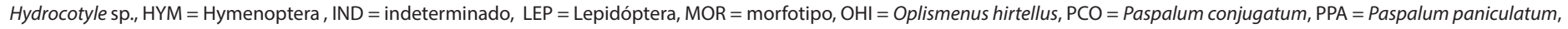
$\mathrm{PEL}=$ pelos, $\mathrm{PIE}=$ piedras, $\mathrm{POL}=$ Polygonum $\mathrm{sp} ., \mathrm{RAV}=$ restos de avena, $\mathrm{SAR}=$ Sorghum arundinaceum . 


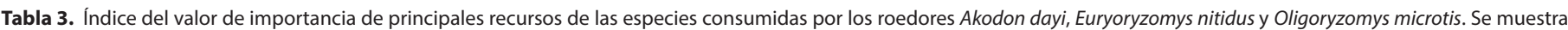

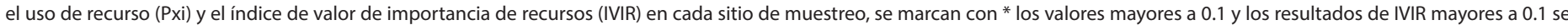
encuentran resaltados con negrillas.

\begin{tabular}{|c|c|c|c|c|c|c|c|c|c|c|c|c|c|c|}
\hline \multirow[t]{2}{*}{ Familia/Orden } & \multirow[t]{2}{*}{ Especie/Morfoespecie } & \multicolumn{4}{|c|}{ SAF simple } & \multicolumn{2}{|c|}{ SAF inter } & \multicolumn{3}{|c|}{ SAF complejo } & \multicolumn{4}{|c|}{ Bosque } \\
\hline & & $\begin{array}{l}\text { O.mi } \\
\text { Pxi }\end{array}$ & $\begin{array}{l}\text { E.ni } \\
\text { Pxi }\end{array}$ & $\begin{array}{c}\text { A.da } \\
\text { Pxi }\end{array}$ & IVIR & $\begin{array}{l}\text { E.ni } \\
\text { Pxi }\end{array}$ & IVIR & $\begin{array}{l}\text { O.mi } \\
\text { Pxi }\end{array}$ & $\begin{array}{l}\text { E.ni } \\
\text { Pxi }\end{array}$ & IVIR & $\begin{array}{c}\text { O.mi } \\
\text { Pxi }\end{array}$ & $\begin{array}{l}\text { E.ni } \\
\text { Pxi }\end{array}$ & $\begin{array}{c}\text { A.da } \\
\text { Pxi }\end{array}$ & IVIR \\
\hline \multicolumn{15}{|l|}{ Dicotyledoneae } \\
\hline Convolvulaceae & Ipomoea sp. & 0.03 & 0.01 & & 0.04 & & & & & & 0.12 & & 0.13 & 0.25 \\
\hline Papilionaceae & Desmodium sp. & & & & & & & & & & & $0.26^{*}$ & & 0.26 \\
\hline Polygonaceae & Polygonum sp. & & & 0.02 & 0.02 & 0.04 & 0.04 & & 0.02 & 0.02 & 0.06 & $0.23^{*}$ & & 0.29 \\
\hline \multicolumn{15}{|c|}{ Monocotyledoneae } \\
\hline Araceae & Anthurium sp. & 0.01 & & & 0.01 & 0.09 & 0.09 & 0.10 & 0.04 & 0.19 & & & 0.02 & 0.02 \\
\hline Cyperaceae & Cyperus chalaranthus & & 0.04 & & 0.04 & $0.16^{*}$ & 0.16 & & 0.03 & 0.03 & & & 0.05 & 0.06 \\
\hline \multirow[t]{6}{*}{ Poaceae } & Axonopus compressus & 0.02 & 0.02 & & 0.03 & & & $0.21^{*}$ & & 0.21 & & & 0.11 & 0.11 \\
\hline & Imperata sp. & & & & & & & $0.17^{*}$ & & 0.19 & & & & \\
\hline & Oplismenus hirtellus & & & 0.03 & 0.03 & 0.04 & 0.04 & $0.17^{*}$ & & 0.17 & & 0.12 & 0.09 & 0.20 \\
\hline & Paspalum conjugatum & 0.06 & & 0.09 & 0.14 & & & & & & $0.23^{*}$ & & 0.04 & 0.26 \\
\hline & Paspalum paniculatum & 0.06 & & $0.16^{*}$ & 0.22 & 0.13 & 0.13 & & & & $0.20^{*}$ & & 0.01 & 0.21 \\
\hline & Paspalum sp. & & $0.23^{*}$ & & 0.24 & 0.04 & 0.04 & & & & & 0.03 & & 0.03 \\
\hline \multicolumn{15}{|l|}{ Glomeromycetes } \\
\hline \multirow[t]{2}{*}{ Glomerales } & Glomus clavisporum & & & & & & & & $0.36^{*}$ & 0.36 & & & & \\
\hline & Glomus sp2. & $0.10^{*}$ & & 0.07 & 0.17 & & & & & & & & & \\
\hline \multicolumn{15}{|l|}{ Insecta } \\
\hline & Coleoptera sp1. & & & 0.07 & 0.07 & & & & & & & & & \\
\hline \multirow[t]{2}{*}{ Coleoptera } & Coleoptera sp4. & & $0.23^{*}$ & & 0.23 & & & & & & & & & \\
\hline & Coleoptera sp5. & & & & & & & & $0.22^{*}$ & 0.22 & & & & \\
\hline
\end{tabular}

SAF simple = Sistema agroforestal simple-monocultivo; SAF inter = Sistema agroforestal intermedio; SAF complejo = Sistema agroforestal complejo; Bosque = Bosque secundario. O.mi = Oligoryzomys microtis; $\mathrm{E} . \mathrm{ni}=$ Euryoryzomys nitidus; $\mathrm{A} . \mathrm{da}=$ Akodon dayi .

consumo. En el SAF complejo O. microtis presentó a los restos vegetales como su primordial categoría alimenticia y E. nitidus a la categoría micorrizas como su recurso principal. Finalmente, en el bosque las tres especies de roedores consumen primordialmente restos vegetales (tallos, hojas, espiguillas entre otros). A pesar de estas diferencias cualitativas en la dieta, las pruebas de Kruskal-Wallis concluyen que no hay diferencias significativas ni entre especies $(H=$ $2.213, g . l .=2, P=0.331)$, ni por sitio de estudio $(H=6.700$, $g . l=3, P=0.082$ ).

Las medidas de sobreposición de nicho trófico mostraron un rango con valores bajos (ISP $=0.11$; IMM $=0.10)$ entre E. nitidus y $A$. dayi; y entre $E$. nitidus y $O$. microtis (ISP $=0.22$; $I M M=0.21$ ). Mientras que, al comparar O. microtis con $A$. dayi se tuvo el mismo valor de solapamiento con ambos índices $(I S P=0.26 ; I M M=0.26)$. Estos valores representan baja sobreposición e indican poca similitud entre los ítems de las dietas de estas especies de roedores y sugieren que no hay competencia por alimento entre ellos.

El análisis del coeficiente de variación (CV) más bajo obtenido fue para $A$. dayi en la categoría restos vegetales monocotiledóneas con $5.4 \%$ y dicotiledóneas $7.3 \%$. Para E. nitidus el CV más bajo fue para la categoría restos vege- tales monocotiledóneas $46.2 \%$; y para O. microtis el CV más bajo fue también para la categoría restos vegetales monocotiledóneas, confirmando así la alta preferencia por estas categorías alimenticias (Tabla 4).

\section{Discusión}

La composición de la dieta mostró que las tres especies de roedores estudiadas consumen restos vegetales. La alta proporción de tejidos vegetales para estas tres especies puede estar asociado a la fácil obtención de energía para su mantenimiento, en comparación con una dieta basada en proteínas (Suárez 1994) y a la disponibilidad del recurso en el ambiente. Es de esperar que estas especies de roedores aprovechen los recursos disponibles de los lugares donde habitan o se refugian. Así, la familia más importante fue Poaceae, encontrándose en los cuatro sitios de muestreo y en la dieta de las tres especies evaluadas (Tabla 3; Apéndice 3), además de varias especies de dicotiledóneas. Sin embargo, no se alimentan exclusivamente de ellos cuando estaban presentes otros ítems disponibles, como micorrizas y artrópodos como se reportó para las tres especies; pero para O. microtis únicamente en SAF simple, aunque estas categorías estaban presentes en SAF complejo y en bosque. 
Tabla 4. Coeficiente de variación (CV) calculado para cada categoría alimenticia en las especies.

\begin{tabular}{|c|c|c|c|c|c|c|c|c|c|}
\hline \multirow{2}{*}{ Especies } & \multicolumn{2}{|c|}{ RVe } & \multirow{2}{*}{ Mic } & \multicolumn{2}{|c|}{ Art } & \multirow{2}{*}{ Sem } & \multirow{2}{*}{ Esp } & \multirow{2}{*}{ T.V.M } & \multirow{2}{*}{ Otros } \\
\hline & Dic & Mon & & Adu & Lar & & & & \\
\hline Oligoryzomys microtis & 1.19 & 0.91 & 1.73 & 1.73 & & & 1.61 & 1.73 & 0.99 \\
\hline Euryoryzomys nitidus & 0.93 & 0.46 & 2.00 & 1.20 & 2.00 & 1.20 & 1.28 & 1.16 & 0.59 \\
\hline Akodon dayi & 0.07 & 0.05 & 0.83 & 0.20 & & & 0.99 & 0.36 & 0.25 \\
\hline
\end{tabular}

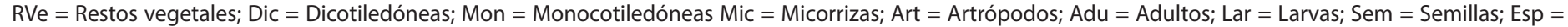
Espiguillas; T.V.M = Tejido Vascular (Morfotipos), Otros.

El género Oligoryzomys es considerado principalmente granívoro en estaciones secas, mientras que en estaciones húmedas su dieta consiste en follaje y ocasionalmente artrópodos (Spotorno et al. 2000). Nuestro estudio no realizó una comparación entre estaciones húmedas o secas; sin embargo, por los altos porcentajes de restos vegetales encontrados la dieta $O$. microtis es considerada herbívora para la época del estudio. Para el caso de Akodon dayi, reportes previos consideran al género Akodon principalmente insectívoro (Vorontsov 1961; Solari 2007; Suarez y Bonaventura 2001; Polop et al. 2013). Sin embargo, la presencia de restos vegetales de dicotiledóneas y monocotiledóneas observadas en $A$. dayi concuerda con reportes para otras especies del género, que complementan su dieta con el consumo de hojas (Pearson y Pearson-Ralph 1978; Brandan 1995; Giannoni et al. 2005; Silva 2005); además nosotros reportamos el consumo de micorrizas Glomus fasciculatum y Glomus sp. pero solamente en bosque.

Respecto al consumo de artrópodos, se evidenciaron mayores valores en el orden Coleoptera para las especies $A$. dayiy E. nitidus y un mayor consumo del orden Hymenoptera (familia Formicidae) en O. microtis. Estudios previos en la dieta de los géneros Euryoryzomys y Oligoryzomys reportaron el consumo de artrópodos Hymenoptera, Orthoptera, Araneae, larvas de Lepidoptera, larvas de Coleoptera e Hymenoptera, entre otros (Pinotti 2005; Siqueira 2013; Cervantes 2014). Reportes previos sugieren que miembros de las tribus de Akodontini (al que Akodon dayi corresponde), y Oryzomyini (que agrupa a Oligoryzomys microtis y Euryoryzomys nitidus, entre otros) consumen primordialmente insectos (Dorst 1972; Pizzimenti y De Salle 1980; Meserve 1981; Ellis et al. 1994; Noblecilla-Huiman 2008; Noblecilla y Pacheco 2012; Polop et al. 2015). Sin embargo, nuestro estudio sugiere que, si bien los artrópodos forman parte de la dieta de estas especies, estos no son elementos exclusivos ni mayoritarios.

En este estudio no se hizo un relevamiento de artrópodos. Sin embargo, de acuerdo con las características de los sistemas de producción, se espera que el SAF simple-monocultivo con sombra temporal, SAF complejo y bosque (con alto porcentaje de sombra a la altura del piso) presenten mayor abundancia de artrópodos. Esto explicaría el elevado porcentaje de artrópodos ingeridos en la dieta de las tres especies de roedores. Cabana (2016) reportó una mayor abundancia de los grupos Coleoptera, Hemiptera e Hymenoptera en sistemas de cultivos tradicionales. En este estudio Coleoptera e Hymenoptera fueron los más consu- midos en SAF simple-monocultivo.

También se reportó la presencia de esporocarpos de micorrizas en las tres especies de roedores sigmodontinos evaluados. Otros estudios reportaron esta categoría en roedores oryzomyinos de la Amazonía (Janos et al. 1995) y en bosques montanos (Noblecilla y Pacheco 2012; Cervantes 2014). En este estudio no se encontraron micorrizas durante la colecta general de plantas; no obstante, al igual que los artrópodos se podría esperar que sitios con un alto porcentaje de sombra, como SAF complejo y bosque, presenten mayor abundancia de hongos. Esto explicaría la mayor cantidad de esporocarpos e hifas encontrados en la dieta de $A$. dayi y $E$. nitidus en dichos sitios, pero no explicaría la presencia de éstos en los restos estomacales de 0 . microtis en SAF simple-monocultivo.

La alta presencia de restos vegetales en la dieta de estas tres especies, puede deberse más a un proceso fisiológico que de dieta, debido a que la tasa de digestibilidad de restos vegetales es menor que el de semillas y frutos. El aparato digestivo de los roedores está adaptado para una alimentación a base de plantas, ya que digieren la celulosa en el ciego, donde se da lugar a la fermentación microbiana; además de la presencia de la cecotrofia, donde expulsan contenidos del intestino a medio digerir y los vuelven a ingerir para hacer una segunda digestión (Megías et al. 2016). Conectar la morfología del estómago con la dieta en los roedores es una práctica común (Vorontsov 1961). Sin embargo, este enfoque reduccionista está poco respaldado por datos y puede conducir a conclusiones sesgadas. Por ello, debe evitarse llegar a conclusiones demasiado generalizadas sin tener en cuenta otras partes importantes del aparato digestivo (Pardiñas et al. 2020). Otros autores concuerdan que la variación en la dieta entre especies está relacionada con la morfología del roedor, debido a que especies más pequeñas (más oportunistas) consumen una dieta rica en energía, principalmente insectos, mientras que especies más grandes en tamaño (menos oportunistas) consumen mayormente vegetación (Pizzimenti y De Salle 1980; Ellis et al. 1994; Noblecilla-Huiman 2008). Sin embargo, en el presente estudio, se pudo ver que la misma especie puede comportarse como más especialista o más generalista, dependiendo de la disponibilidad de alimento en al ambiente aprovechando incluso, ítems más proteolíticos (insectos) cuando éstos están presentes.

Respecto a la presencia de semillas en la dieta, no se detectaron en el aparato digestivo de las especies estudiadas (aunque sí se determinó su presencia en los diferentes cultivos muestreados). Sólo se reportó la presencia de semi- 
Ilas de Polygonum sp. en E. nitidus en SAF intermedio y SAF complejo, a pesar de que reportes previos para el género lo categorizan como frugívoro-granívoro (Pinotti et al. 2011; Paglia et al. 2012).

La dieta poco amplia se relaciona con el valor de importancia del recurso, que a su vez estaría relacionada con el grado de perturbación del sitio, esperando una mayor disponibilidad de recursos alimenticios en los sistemas agroforestales (SAFs) intermedios/complejos de cacao (perturbación intermedia; Connell 1978). La evaluación de los hábitos alimenticios mediante la amplitud de nicho trófico mostró que, tanto A. dayi como O. microtis, tienen una dieta amplia en SAF simple-monocultivo y bosque y menos amplia en SAF complejo para O. microtis, debido a que sólo se hallaron restos vegetales en sus contenidos estomacales.

Sin embargo, para $E$. nitidus se observó una dieta amplia en SAF simple-monocultivo y SAF complejo; mientras que, en parcelas SAF intermedio y bosque, E. nitidus presenta una menor amplitud trófica, catalogándose como especialista. Estos resultados no encajarían con la predicción realizada; teniendo que ser respuestas conductuales y de preferencia de recursos los que explique estos resultados. Así, en SAF simple-monocultivo y SAF complejo, estaría aprovechando los recursos disponibles cercanos a sus refugios sin invertir mucho gasto energético para su obtención. Por otro lado, los bajos valores obtenidos por la amplitud de nicho trófico en E. nitidus se explican con la dieta especializada en la familia Cyperaceae en el SAF intermedio y una dieta basada en la familia Papilionaceae y Polygonaceae en el bosque (Tabla 3), aunque estos resultados deben ser tomados con cuidado, ya que solo se pudieron evaluar dos individuos de esta especie en cada tipo de hábitat. Por ello, los resultados obtenidos pueden ser reflejo más de una "preferencia individual" o una característica del grupo.

Las medidas de sobreposición de nicho presentaron un rango con valores bajos entre las tres especies lo que sugiere que no hay competencia por los recursos entre ellas, o que, debido a una competencia interespecífica, se presenta una diferenciación de los ítems consumidos. Sin embargo, debido al bajo número de capturas de especies por tipo de cultivo, no se pudo diferenciar la dieta por hábitat. Un análisis con mayor número de muestras es necesario para confirmar nuestros resultados.

La dieta de estas tres especies de roedores, para el periodo de transición seca-húmeda en Alto Beni es diversa, y la selección de su alimento esta posiblemente relacionada con la disponibilidad de los recursos alimenticios presentes en cada tipo de parcela. SAF simple-monocultivo presenta una mayor cantidad de plantas herbáceas a nivel del suelo, a diferencia de SAF complejo y bosque, donde al haber una mayor proporción de hojarasca, también hay una menor proporción de hierbas. Según Reichman (1977) la selección nutricional es uno de los factores que determina las preferencias dietéticas en los roedores. Así, considerando los requerimientos energéticos y nutricionales de estas especies, la disponibilidad de ítems energéticamente más rentables (tallos y hojas de dicotiledóneas y monocotiledóneas) parece ser la responsable de un mayor o menor nicho trófico. Por lo tanto, podemos concluir que estas tres especies de roedores presentan diferentes tipos de hábitos alimenticios dependiendo del ambiente donde se encuentran: O. microtis es herbívoro-omnívoro, se califica como generalista en parcelas SAF simple-monocultivo y en el bosque y menos generalista en SAF complejo. Euryoryzomys nitidus es predominante herbívoroomnívoro, se califica como generalista en parcelas SAF simple-monocultivo y menos generalista en SAF complejo; especialista en parcelas SAF intermedio y en el bosque intervenido. Akodon dayi presenta una dieta herbívorainsectívora, es considerada generalista en parcelas SAF simple-monocultivo y en el bosque intervenido.

Nuestro estudio señala que la dieta de estos roedores depende de la disponibilidad de alimento encontrado en su hábitat. Sin embargo, cuando existan otros recursos disponibles estos aprovecharan para incluirlos en su dieta, presentando un comportamiento oportunista con relación a la presencia de ítems alimenticos. Se deben profundizar estudios de dieta de otras especies de roedores, realizando muestreos estacionales en sistemas agroforestales, tomando en cuenta épocas reproductivas, sexo y edad para comparar con los resultados obtenidos en este estudio.

\section{Agradecimientos}

Un reconocimiento y agradecimiento especial al Dr. Sydney Anderson, por el trabajo desarrollado en Bolivia y haber impulsado no solo el conocimiento de los roedores de Bolivia, sino por haber iniciado escuela en ese tema. Agradecemos al proyecto" ¿Los sistemas agroecológicos más complejos conservan mejor las funciones ecosistémicas?, caso del estudio de la relación herbívoro - depredador en cultivos de cacao en Alto Beni" por el financiamiento brindado para que este estudio pueda realizarse. A la Colección Boliviana de Fauna (CBF) por el apoyo con material y al Herbario Nacional de Bolivia (HNB) por su contribución en la identificación de especies vegetales. El valioso apoyo de L. Marconi- Ripa por su colaboración en la identificación de muestras vegetales, a J. M. Limachi por su colaboración en la identificación de invertebrados. A S. Revollo, I. Molina, D. Rivas por su colaboración y motivación en el trabajo de campo. Por último, agradecemos el trabajo editorial de tres revisores anónimos que contribuyeron a mejorar versiones previas de este manuscrito y la invitación a participar en este número conmemorativo.

\section{Literatura Citada}

Aguirre, L. F., T. Tarifa, R. B. Wallace, N. Bernal, L. Siles, E. Aliaga-Rossel, y J. Salazar-Bravo. 2019. Lista actualizada y comentada de los mamíferos de Bolivia. Ecología en Bolivia 54:107-147.

Amaya, M. 1991. Interacción Planta- Colibrí en Macayacu (Amazonas, Colombia): Una perspectiva palinológica. Caldasia 23:301-322. 
Anderson, S., y T. Tarifa. 1996. Los Mamíferos Endémicos de Bolivia. Ecología en Bolivia 28:45-64.

Anderson, S. 1997. Mammals of Bolivia: Taxonomy and distribution. Bulletin of the American Museum of Natural History. New York 231:1-652.

Aubry, K. B., J. P. Hayes, B. L. Biswell, y B. G. Marcot. 2003. The ecological role of three dwelling mammals in western coniferous forest. Pp. 415-443 in Management and conservation in the forest of western North America (Zabel, C. J., y R. G. Anthony, eds.). Cambridge University. Cambridge, Inglaterra

Bazoberry, O. C. y C. C. Salazar. 2008. El cacao en Bolivia: una alternativa económica de base campesina indígena. CIPCA. La Paz, Bolivia.

Beer, J., R. Muschler, D. Kass, y E. Somarriba. 1998. Shade management in coffee and cacao plantations. Agroforestry Systems 38:139-164.

BLum, M. 2011. Las Parcelas Familiares de las Comunidades Interculturales del Alto Beni: Una Propuesta Técnica para la Soberanía Alimentaria y Reducir el Cambio Climático de Bolivia. Fundación Renace. La Paz, Bolivia.

Brandan, Z. J. 1995. Contribución al conocimiento de la dieta de Akodon simulator simulator. Acta Zoológica Lilloana 43:73-79.

Brown, J. S. 2000. Foraging ecology of animals in response to heterogeneous environments. Pp.181-214 in The ecological consequences of environmental heterogeneity, Chapter 10 (Hutchings, J., y A. Stewart) Blackwell Science Ltd. Oxford, Inglaterra.

Cabana, A. G. 2016. Diversidad de artrópodos en tres sistemas de cultivos de cacao (Theobroma cacao L.) en la región Ucayali- Perú. Licenciatura en Ingeniería Agronómica. Universidad Nacional de San Agustín de Arequipa.

Campos, C. M., S.M. Giannoni, P. Taraborelli, y C. E. Borghi. 2007. Removal of mesquite seeds by small rodents in the Monte desert, Argentina. Journal of Arid Environments 69:228-236.

Centro agronómico tropical de investigación y enseñanza (CATIE). 2002. Proyecto modernización de la cacaocultura orgánica de Alto Beni, Bolivia. Turriabla, Costa Rica.

Cervantes, O. K. 2014. Dieta de roedores Sigmodontinos (Rodentia: Cricetidae) en los bosques montanos del Valle del Río Holpas Ayacucho, Perú. Licenciatura en Biología. UNMSM de Perú.

ConnelL, J. H. 1978. Diversity in tropical rain forest and coral reefs. Science 199:1302-1310.

Cortés, A., J. R. Rau, E. Miranda, y J. E. Jiménez. 2002. Hábitos alimenticios de Lagidium viscacia y Abrocoma cinerea: roedores sintópicos en ambientes altoandinos del norte de Chile. Revista Chilena de Historia Natural 75:583-593.

De la Cruz-Ramírez, A., y S. Sánchez-Soto. 2016. Estructura poblacional de roedores plaga en caña de azúcar (Saccharum spp.) en la Chontalpa, Tabasco, México. Agroproductividad 9:35-40.

Dobson, A., y J. Foufopoulos. 2001. Emerging infectious pathogens of wildlife. Philosophical transactions of the Royal Society of London. Series B, Biological Sciences 356: 1001-12.

Dorst, J. 1972. Morphologie de estomac t regime alimentaire de quelques rongeurs deshautes Andes du Perou. Mammalia 36:647-656
Dytham, C. 2001. Choosing and using Statistics: a biologist guide, 2da ed. Wiley Blackwell Press. York, Inglaterra.

EisenberG, J. F., y K. H. Redford. 1999. Mammals of the Neotropics. The Central Neotropics: Ecuador, Peru, Bolivia, Brazil. University of Chicago Press. Chicago, EE.UU.

Ellis, B. A., J. Mills, E. Kennedy, J. Maiztegui, y J. Childs. 1994. The relationship among diet, alimentary tract morphology, and life history for five species of rodents from the central Argentine pampa. Acta Theriologica 39:345-355.

Emmons, L. H., y F. Feer. 1997. Neotropical rainforest mammals: a field guide. Chicago: The University of Chicago Press. Chicago, EE.UU.

Estrada, A., y R. Coates-Estrada. 2005. Diversity of Neotropical migratory landbird species assemblages in forest fragments and man-made vegetation in Los Tuxtlas, Mexico. Biodiversity and Conservation 14:1719-1734.

Giannoni, S. M., C. Borghi, M. Dacar, y C. M. Campos. 2005. Main food categories in diets of sigmodontine rodents in the monte Argentina. Neotropical mammozoology 12:181-187.

Guabloche, A., M. Arana, y O. E. Ramírez. 2002. Diet and gross gastric morphology of Oryzomys xantheolus (Sigmodontinae, Rodentia) in Peruvian Loma. Mammalia 66:405-411.

HolecheK, J. L., y M. Vavra. 1981. The effect of slide and frequency observation numbers on the precision of microhistological analysis. Journal of Range Management 34:337-338.

HolecheK, J. L., M. Vavra, y R. D. Pieper. 1982. Botanical composition determination of range herbivore diet: $A$ Review. Journal of Range Management 35:309-315.

Horn, H. S. 1966. Measurement of "overlap" in comparative ecological studies. American Naturalist 100:419-424.

Hughes, J. B., G. C. Daily, y P. R. Ehrlich. 2002. Conservation of tropical forest birds in countryside habitats. Ecology Letters 5:21-129.

Janos, D., C. T. Sahley, y L. H. Emmons. 1995. Rodent dispersal of vesicular-arbuscular mycorrhizal fungi in Amazonian Perú. Ecology 76:1852-1858.

July-Martínez, W. 2007. Caracterización morfológica y molecular del Cacao Nacional Boliviano y de selecciones élites del Alto Beni, Bolivia. Tesis de maestría. Escuela de Posgrado, Programa de Educación para el Desarrollo y la Conservación del Centro Agronómico Tropical de Investigación y Enseñanza (CATIE). Turrialba, Costa Rica.

Killeen, T. J., T. M. Siles, L. Soria, y L. Correa. 2005. Estratificación de vegetación y cambio de uso de suelo en los Yungas y Alto Beni de La Paz. Ecología en Bolivia 40: 32-69.

Killeen, T. J., V. Calderon, L. Soria, B. Quezada, M. K. Steininger, G. Harper, L. A. Solórzano, y C. J. Tucker. 2007. Thirty years of land-cover change in Bolivia. AMBIO 36:600-606.

KREBS, C. J. 1985. Ecology the experimental analysis of distribution and abundance. 2da ed. Addison Wesley Longman Press. Menlo Park, EE.UU.

LeVINS, R. 1968. Evolution in changing environments: Some theoretical explorations. Princeton University. New Jersey, EE.UU.

López-Cortés, F., A. Cortés, E. Miranda, y J. Rau. 2007. Dietas de Abrothrix andinus, Phyllotis xanthopygus (Rodentia) y Lepus europaeus (Lagomorpha) en un ambiente altoandino de Chile. Revista Chilena de Historia Natural 80:3-12. 
LóPEz, T. G. 2007. Sistemas agroforestales 8. SAGARPA. Subsecretaría de Desarrollo Rural. Colegio de Post-graduados. Puebla, México.

Maguiña, N. 2011. Dieta de murciélagos filostómidos (Chiroptera: Phyllostomidae) del valle de Kosñipata, San Pedro, Cuzco, Perú. Tesis para optar el grado de Bióloga. Universidad Peruana Cayetano Heredia.

Megías, M., P. Molist, y M. A. Pombal. 2016. Atlas de histología vegetal y animal. Órganos animales-sistema digestivo. Disponible en: https://mmegias.webs.uvigo.es/descargas/oategumento.pdf. Consultado el 15 de junio de 2018.

Mercado, I. 1991. Dos roedores simpátricos en el Valle de La Paz: análisis de la morfología molar y la dieta. Ecología en Bolivia 18:28-43.

Meserve, P. L. 1981. Trophic relationship among small mammals in a Chilean semiarid thorn scrub community. Journal of Mammalogy 62:304-314.

Mızz, J. 2010. Producción de Naranja (Citrus sinensis) en sistemas agroforestales sucesionales en Alto Beni, Bolivia - Estudio de caso. Pp. 324-340 in Biodiversidad y Ecología en Bolivia (Beck S., ed.). Instituto de Ecología. Universidad Mayor de San Andrés. La Paz, Bolivia.

Monge, J. 2007. ¿Qué son plagas vertebradas? Agronomía Costarricense 31:111-121.

Montero, S. H., y A. Gonzáles. 2017. ¿Los roedores, enemigos o aliados de los ecosistemas? INECOL. Eco-Lógico. México. Disponible en: http://www.inecol.mx/inecol/index.php/es/ctmenu-item-25/ct-menu-item-27/462-losroedores-enemigos-oaliados-de-los-ecosistemas.pdf. Consultado el 15 de junio de 2018.

Musser, G. G., M. D. Carleton, E. M. Brothers, y A. L. Gardner. 1998. Systematic studies of oryzomyine rodents (Muridae: Sigmodontinae): diagnoses and distributions of species formerly assigned to Oryzomys "capito". Bulletin of the American Museum of Natural History 236:1-376.

Musser, G. G., y M. D. Carleton. 2005. Superfamily Muroidea. Pp. 501-755 in Mammal Species of the World: A Taxonomic and Geographic Reference (Wilson, D. E., y D. M., Reeder, eds.). The Johns Hopkins University Press. Baltimore, EE.UU.

Myers, P., J. Patton, y M. Smith. 1990. A Review of the Boliviensis group of Akodon (Muridae: Sigmodontinae), with emphasis on Peru and Bolivia. Miscellaneous Publications Museum of Zoology University of Michigan 177: 1-104.

Navarro, G., y M. Maldonado. 2002. Geografía ecológica de Bolivia. Vegetación y Ambientes Acuáticos. Editorial Centro de Ecología Simón I. Patiño. Cochabamba, Bolivia.

Navarro, G. 2011. Clasificación de la vegetación de Bolivia. Editorial Centro de Ecología Difusión Simón I. Patiño. Santa Cruz de la Sierra, Bolivia.

Navia, J. F. 2000. Agroforestería. Actualización profesional en manejo de recursos naturales, agricultura sostenible y pobreza rural. Corporación Colombiana de Investigación Agropecuaria, Centro para el Desarrollo Agropecuario y Forestal Inc. (CEDAF). Santo Domingo, República Dominicana.

Noblecilla, M. C., y V. Pacheco. 2012. Dieta de roedores sigmodontinos (Cricetidae) en los bosques montanos tropicales de Huánuco, Perú. Revista Peruana de Biología 19:313-318.

Noblecilla-Huiman, M. C. 2008. Dieta y morfología del estómago en roedores de los bosques montanos del departamento de Huánuco, Perú. Tesis para optar el grado de Bióloga. Universidad Nacional Mayor de San Marcos.

Osco, F. O. 2000. Estructura y dieta de la comunidad de roedores pequeños en función del borde de bosque y su relación con la regeneración de bosques yungueños quemados, en los alrededores de Chulumani, La Paz, Bolivia. Licenciatura en Biología. Universidad Mayor de San Simón.

Ortiz-Gonzáles, M. 2006. Conocimiento local y decisiones de los productores de Alto Beni, Bolivia, sobre el diseño y manejo de la sombra en sus cacaotales. Tesis de magister scientiae. Programa de Educación para el Desarrollo y la Conservación de Costa Rica.

Paglia, A. P., G. Da Fonseca, A. Rylands, G. Herrmann, L. Aguiar, A. Chiarello, Y. Leite, L. Costa, S. Siciliano, M. Kierulff, S. Mendes, V. Tavares, R. Mittermeier, y J. Patton. 2012. Lista anotada dos mamíferos do Brasil. 2da ed. Arlington: Conservation International.

Pardiñas U. F. J., P. Teta, D. Alvarado- Serrano, L. Geise, J. P. Jayat, P. E. Ortiz, P. R. Gonçalves, y G. D'Elía. 2015. Genus Akodon Meyen, 1833. Pp. 144-204 in Mammals of South America, Volume 2. Rodents (Patton J. L., U. F. J. Pardiñas, y G. D’Elía, eds.). The University of Chicago Press. Chicago, EE.UU.

PardiñasU.F.J., C. Cañón, C.A.Galliari,J. Brito, N. Bernal Hoverud, G. Lessa, J. A. DE OliveiRA. 2020. Gross stomach morphology in Akodontine rodents (Cricetidae: Sigmodontinae: Akodontini): a reappraisal of its significance in a phylogenetic context. Journal of Mammalogy 101:835-857

Patton, J. L., M. N. F. da Silva, y J. R. Malcolm. 2000. Mamíferos del Rio Juruá y la diversificación evolutiva y ecológica en Amazonia. Boletín del Museo Americano de Historia Natural 244:1-306.

Patton, J. L., U. F. Pardiñas, y G. D’Elia (eds.). 2015. Mammals of South America, Volume 2: Rodents. University of Chigaco Press. Chicago, EE.UU.

Percequillo A. R. 2015. Genus Euryoryzomys Weksler, Percequillo, and Voss, 2006. Pp. 321-321 in Mammals of South America, Volume 2. Rodents (Patton J. L., U. F. J. Pardiñas, y G. D'Elía, eds.). The University of Chicago Press. Chicago, EE.UU. Peñafiel, R. M. 2000. Uso y manejo de plaguicidas y el efecto del viento en la contaminación de bordes en aéreas del cultivo del Alto Beni, La Paz, Bolivia. Tesis de Maestría. Facultad Agronomía. Universidad Mayor de San Andrés.

Pearson, O. P., y C. P. Pearson-Ralph. 1978. The diversity and abundance of vertebrates along an altitudinal gradient in Peru. Memorias del Museo de Historia Natural "Javier Prado" Perú 18:1-97.

Pianka, E. R. 1973. The structure of lizard communities. Annual Review of Ecology and Systematics 4:53-74.

Pinotrı, B. T. 2005. Dieta e influencia da disponibilidade de alimento na distribuicao espacial de species de pequenos mamíferos em uma área de Mata Atlantica madura do Estado de Sao Paulo. Instituto de Biociencias da Universidade de Sao Paulo. Sao Paulo.

Pinotti, B. T., L. Naxara, y R. Pardini. 2011. Diet and food selection by small mammals in an old-growth Atlantic forest of south-eastern Brazil. Neotropical Fauna and Environment 46:1-9.

Pizzimenti, J. J., y R. De Salle. 1980. Dietary and morphometric variation in some Peruvian rodent communities: the effect 
of feeding strategy on evolution. Biological Journal of the Linnean Society 13:263-285.

Polop, F., L. Sepúlveda, A. Pelliza-Sbriller, J. Polop, y M.C. Provenzal. 2015. Estructura de la dieta de roedores sigmodontinos en arbustales del ecotono bosque-estepa del suroeste de Argentina. Mastozoología Neotropical 22:85-95.

Polop, F., A. Pelliza Sbriller, L. Sepulveda, M. C. Provenzal, Y J. Polop. 2013. Hábitos alimentarios de roedores sigmodontinos en hábitats de matorral modificados del sur-oeste de Argentina. Libro de Resúmenes. XXVI Jornada argentinas de Mastozoología. Mar del Plata, Argentina.

Poleo, C. J., L. Rodríguez, y J. Garbi. 2006. Métodos alternativos para el control de ratas en el cultivo arroz. CENIAP Hoy 11:7.

Pons, J., y J. G. Pausas. 2007. Acorn dispersal by radio-tracking. Oecologia 153: 903-911.

Reichman, O. J. 1977. Optimization of diets through food preferences by heteromyid rodents. Ecology 58:454-457.

Silva, S. 2005. Posiciones tróficas de pequeños mamíferos en Chile: Una revisión. Revista Chilena de Historia Natural 78:589-599.

Sikes, R. S., y the Animal Care and use committee of the american SOCIETY OF MAMmalogists. 2016. Guidelines of the American Society of Mammalogists for the use of wild mammals in research and education. Journal of Mammalogy 97:663-688.

Siqueira, R. 2013. Historia natural y ecología de duas espécies de roedores simpátricas da tribo Oryzomyini (Cricetidae: Sigmodontinae) na Floresta Atlántica. Tese doutorado. Escola Superior de Agricultura "Luiz de Queiroz". Centro de Energia Nuclear na Agricultura.

SolaRI, S. 1997. Relaciones tróficas en una comunidad de roedores altoandinos en el Parque Nacional Manu. Licenciatura en Biología. UNMSM de Perú.

SolaRl, S. 2007. Trophic relationships within a Highland rodent assemblage from Manu National Park, Cusco, Peru. in The Quintessential Naturalist: Honoring the Life and Legacy of Oliver (Kelt, D., E. P. Lessa, J. Salazar-Bravo, y J. L. Patton, eds.). Pearson. University of California Publications in Zoology 134: 225-240

SomarRiBA, E., y L. Trujillo. 2005. El Proyecto“Modernización de la cacaocultura orgánica del Alto Beni, Bolivia". Agroforestería en las Américas 43-44.

SuAREz, O. V. 1994. Diet and habitat selection of Oxymycterus rutilans (Rodentia, Cricetidae). Mammalia 58:225-234.

Suarez, O. V., y S. M. Bonaventura. 2001. Habitat use and diet in sympatric species of rodents of the low Parana delta, Argentina. Mammalia 65:167-176.

Spotorno, A. E., R. E. Palma, y J. P. Valladares. 2000. Biología de roedores reservorios de hantavirus en Chile. Revista chilena de infectología 17:197-210.

Tarifa, T., y E. Yensen. 2001. Mamíferos de los bosques de Polylepis de Bolivia. Ecología en Bolivia 9:29-44.

Traveset, A. 1998. Effect of seed passage through vertebrate frugivores guts on germination: a review. Perspectives in Plant Ecology, Evolution and Systematics 1:151-190.

Tzab-Hernández, L. A. y M.C. Macswiney. 2014. Roedores ¿plagas indeseables o animales útiles? CONABIO. Biodiversitas 115:12-16.

Vázquez, L. B. 1997. Dieta y demografía de una comunidad de pequeños roedores en dos hábitats contrastantes. Tesis de licenciatura. Universidad de Guadalajara. División de Ciencias Ambientales. Guadalajara, México.

Vázquez, L. B., G. N. Cameron, y R. A. Medellín. 2000. Hábitos alimentarios y biología poblacional de dos especies de roedores en el occidente de México. Revista Mexicana de Mastozoología 4:5-21.

Veloso, C., y F. Bozinovic. 2000. Effect of food quality on the energetics of reproduction in a precocial rodent, Octodon degus. Journal of Mammalogy 81:971-978.

VEGA, M. M. 2005. Planificación agroforestal participativa para el enriquecimiento de fincas cacaoteras orgánicas con especies leñosas perennes útiles en el Alto Beni Bolivia. CATIE. Turrialba, Costa Rica.

Villegas, R., y C. Astorga. 2005. Caracterización morfológica del cacao nacional del Alto Beni, Bolivia. Agroforestería en las Américas 43-44.

Vorontsov, N. N. 1961. Variation in the transformation rates of organs of the digestive systems in rodents and the principle of functional compensation. Evolutionary Morphology 49-52.

Weksler M., y C. R. Bonvicino. 2015. Genus Oligoryzomys Bangs, 1900. Pp 417-437 in Mammals of South America, Volume 2. Rodents (Patton J. L., U. F. J. Pardiñas, y G. D'Elía, eds.). The University of Chicago Press. Chicago, EE.UU.

XIAO, Z. S., P. A. JANSEN, y Z. Zhang. 2006. Using seed-tagging methods for assessing post-dispersal seed fate in rodentdispersed trees. Forest Ecology and Management 223:18-23.

Associated editor: Jorge Salazar Bravo

Submitted: April 10, 2020; Reviewed: April 24, 2020;

Accepted:September 18, 2020; Published on line: September 26, 2020. 


\section{Apéndice 1}

Lista de especies de plantas colectadas en la zona de estudio, incluyendo el número de individuos colectados y su porcentaje relativo. Se presenta el número de plantas colectadas en cada tipo de sistema agroforestal y bosque.

\begin{tabular}{|c|c|c|c|c|c|c|c|}
\hline Familia & Especie & $\begin{array}{c}\text { Forma de } \\
\text { crecimiento }\end{array}$ & $\begin{array}{c}\text { No total de individuos } \\
\text { colectados }(\%)\end{array}$ & SAF Simple & $\begin{array}{c}\text { SAF } \\
\text { intermedio }\end{array}$ & $\begin{array}{c}\text { SAF } \\
\text { complejo }\end{array}$ & Bosque \\
\hline Alstroemeriaceae & Bomarea sp. & Herbácea & $5(0.21)$ & $5(0.93)$ & & & \\
\hline \multirow[t]{4}{*}{ Amaranthaceae } & Iresine sp. & Arbustiva & $11(0.47)$ & & $4(0.76)$ & $7(1.17)$ & \\
\hline & Iresine diffusa & Herbácea & $22(0.93)$ & & & & $22(3.14)$ \\
\hline & Alternanthera flavescens & Herbácea & $12(0.51)$ & & & & $12(1.71)$ \\
\hline & Amaranthaceae sp. & Herbácea & $22(0.93)$ & $22(4.09)$ & & & \\
\hline \multirow[t]{2}{*}{ Araceae } & Philodendron sp. & Herbácea & $2(0.08)$ & & $2(0.38)$ & & \\
\hline & Anthurium sp. & Herbácea & $24(1.02)$ & & $8(1.53)$ & $16(2.68)$ & \\
\hline Araliaceae & Hydrocotyle sp. & Herbácea & $12(0.51)$ & $12(2.23)$ & & & \\
\hline Arecaceae & Astrocaryum murumuru & Arbórea (plántula) & $8(0.34)$ & & & & $8(1.14)$ \\
\hline Asclepiadaceae & Asclepiadaceae sp. & Herbácea & $36(1.53)$ & & $24(4.60)$ & $12(2.01)$ & \\
\hline \multirow[t]{4}{*}{ Asteraceae } & Bidens andicola & Herbácea & $48(2.04)$ & $12(2.23)$ & & & $36(5.14)$ \\
\hline & Conyza bonariensis & Herbácea & $141(5.98)$ & $25(4.65)$ & $21(4.02)$ & $44(7.36)$ & $51(7.29)$ \\
\hline & Eupatorium sp. & Herbácea & $104(4.41)$ & $18(3.35)$ & $30(5.75)$ & $36(6.02)$ & $20(2.86)$ \\
\hline & Pseudelephantopus spiralis & Herbácea & $27(1.15)$ & $27(5.02)$ & & & \\
\hline Blechnaceae & Blechnum sp. & Helecho & $9(0.38)$ & $4(0.74)$ & $5(0.96)$ & & \\
\hline Commelinaceae & Callisia repens & Rastrera & $18(0.76)$ & $18(3.35)$ & & & \\
\hline Convolvulaceae & Ipomoea sp. & Herbácea & $11(0.47)$ & $2(0.37)$ & & & $9(1.29)$ \\
\hline Costaceae & Costus scaber & Herbácea & $55(2.33)$ & $12(2.23)$ & $14(2.68)$ & $29(4.85)$ & \\
\hline \multirow[t]{3}{*}{ Cyperaceae } & Cyperus chalaranthus & Herbácea & $96(4.07)$ & & $31(5.94)$ & $65(10.87)$ & \\
\hline & Cyperus friburgensis & Herbácea & $57(2.42)$ & $22(4.09)$ & & & $35(5.00)$ \\
\hline & Cyperus sp. & Herbácea & $34(1.44)$ & $8(1.49)$ & $26(4.98)$ & & \\
\hline Dioscoreaceae & Dioscorea sp. & Liana & $15(0.64)$ & & & & $15(2.14)$ \\
\hline \multirow[t]{5}{*}{ Euphorbiaceae } & Acalyphasp. & Herbácea & $13(0.55)$ & $13(2.42)$ & & & \\
\hline & Acalypha stricta & Herbácea & $32(1.36)$ & $17(3.16)$ & & & $15(2.14)$ \\
\hline & Acalypha arvensis & Herbácea & $85(3.60)$ & $19(3.53)$ & $13(2.49)$ & $21(3.51)$ & $32(4.57)$ \\
\hline & Alchornea sp. & Arbórea (plántula) & $5(0.21)$ & & $3(0.57)$ & $2(0.33)$ & \\
\hline & Cnidoscolus sp. & Arbustiva & $9(0.38)$ & & $3(0.57)$ & $6(1.00)$ & \\
\hline \multirow[t]{4}{*}{ Fabaceae } & Bauhinia sp. & Arbórea (plántula) & $11(0.47)$ & & $2(0.38)$ & $3(0.50)$ & $6(0.86)$ \\
\hline & Kudan pueraria phaseoloides & Herbácea & $53(2.25)$ & $16(2.97)$ & $37(7.09)$ & & \\
\hline & Senna sp. & Arbustiva & $6(0.25)$ & & $3(0.57)$ & $3(0.50)$ & \\
\hline & Schizolobium parahypa & Arbórea (plántula) & $4(0.17)$ & & $2(0.38)$ & $2(0.33)$ & \\
\hline Iridaceae & Iridaceae sp. & Herbácea & $67(2.76)$ & & $25(4.79)$ & $42(7.02)$ & \\
\hline Malpighiaceae & Stigmaphyllon cardiophyllum & Liana & $16(0.66)$ & $16(2.97)$ & & & \\
\hline \multirow[t]{3}{*}{ Malvaceae } & Theobroma cacao & Arbórea (plántula) & $17(0.70)$ & $7(1.30)$ & $4(0.77)$ & $6(1.00)$ & \\
\hline & Sida rhombifolia & Arbustiva & $11(0.47)$ & $4(0.74)$ & & & $7(1.00)$ \\
\hline & Sterculia apetala & Arbórea (plántula) & $6(0.25)$ & & & & $6(0.86)$ \\
\hline Melastomataceae & Melastomataceae sp. & Herbácea & $42(1.78)$ & & & & $42(6.00)$ \\
\hline Myrtaceae & Myrcia sp. & Arbustiva & $3(0.13)$ & $3(0.56)$ & & & \\
\hline Papilionaceae & Desmodium sp. & Herbácea & $144(6.11)$ & $15(2.79)$ & $31(5.94)$ & $16(2.68)$ & $82(11.71)$ \\
\hline Phytolaccaceae & Petiveria sp. & Arbustiva & $4(0.17)$ & & & & $4(0.57)$ \\
\hline Piperaceae & Piper aduncum & Arbórea (plántula) & $4(0.17)$ & $1(0.19)$ & & & $3(0.43)$ \\
\hline Plantaginaceae & Plantago sp. & Herbácea & $17(0.72)$ & $17(3.16)$ & & & \\
\hline \multirow[t]{3}{*}{ Poaceae } & Axonopus compressus & Herbácea & 45 (1.91) & & $23(4.41)$ & $22(3.68)$ & \\
\hline & Imperata sp. & Herbácea & $84(3.56)$ & $20(3.72)$ & $27(5.17)$ & $37(6.19)$ & \\
\hline & Leptochloa virgata & Herbácea & $76(3.22)$ & $32(5.95)$ & & & 44 (6.29) \\
\hline
\end{tabular}


Apéndice 1 continuación...

\begin{tabular}{|c|c|c|c|c|c|c|c|}
\hline Familia & Especie & $\begin{array}{c}\text { Forma de } \\
\text { crecimiento }\end{array}$ & $\begin{array}{c}\text { No total de individuos } \\
\text { colectados (\%) }\end{array}$ & SAF Simple & $\begin{array}{c}\text { SAF } \\
\text { intermedio }\end{array}$ & $\begin{array}{c}\text { SAF } \\
\text { complejo }\end{array}$ & Bosque \\
\hline & Oplismenus hirtellus & Herbácea & $75(3.18)$ & & $25(4.79)$ & $50(8.36)$ & \\
\hline & Olyra sp. & Herbácea & $56(2.37)$ & & $30(5.75)$ & $26(4.35)$ & \\
\hline & Paspalum conjugatum & Herbácea & $21(0.89)$ & $21(3.90)$ & & & \\
\hline & Paspalum paniculatum & Herbácea & $154(6.53)$ & $35(6.51)$ & $27(5.17)$ & $55(9.20)$ & $37(5.29)$ \\
\hline & Paspalum sp. & Herbácea & $15(0.64)$ & & $15(2.87)$ & & \\
\hline & Sorghum arundinaceum & Herbácea & $53(2.25)$ & & & & $53(7.57)$ \\
\hline & Poaceae sp1. & Herbácea & 47 (1.99) & $16(2.97)$ & $31(5.94)$ & & \\
\hline & Poaceae sp2. & Herbácea & $20(0.85)$ & $20(3.72)$ & & & \\
\hline \multirow[t]{3}{*}{ Polygonaceae } & Polygonum sp. & Herbácea & $153(6.49)$ & $13(2.42)$ & $23(4.41)$ & $75(12.54)$ & $42(6.00)$ \\
\hline & Triplaris americana & Arbórea (plántula) & $5(0.21)$ & & & & $5(0.71)$ \\
\hline & Campyloneurum sp. & Helecho & $30(1.27)$ & $9(1.67)$ & $12(2.30)$ & $9(1.51)$ & \\
\hline Pteridaceae & Adiantum sp. & Helecho & $35(1.48)$ & $7(1.30)$ & $8(1.53)$ & $5(0.84)$ & $15(2.14)$ \\
\hline Ranunculaceae & Thalictrum sp. & Herbácea & $30(1.27)$ & & & & $30(4.29)$ \\
\hline Rubeacea & Borreria sp. & Herbácea & $10(0.42)$ & $10(1.86)$ & & & \\
\hline \multirow[t]{2}{*}{ Sapindaceae } & Serjania deltoidea & Liana & $30(1.27)$ & $9(1.67)$ & & & $21(3.00)$ \\
\hline & Paullinia alata & Liana & $28(1.19)$ & & & & $28(4.00)$ \\
\hline \multirow[t]{3}{*}{ Solanaceae } & Cestrum sp. & Arbustiva & $5(0.21)$ & & $2(0.38)$ & $3(0.50)$ & \\
\hline & Lycianthes asarifolia & Herbácea & $12(0.51)$ & $12(2.23)$ & & & \\
\hline & Solanum sp. & Arbustiva & $7(0.30)$ & $4(0.74)$ & & & $3(0.43)$ \\
\hline \multirow[t]{2}{*}{ Thelypteridaceae } & Macrothelypteris torresiana & Helecho & $15(0.64)$ & $7(1.30)$ & & & $8(1.14)$ \\
\hline & Thelypteris sp. & Helecho & $8(0.34)$ & & $4(0.77)$ & $4(0.67)$ & \\
\hline Tiliaceae & Triumfetta sp. & Arbustiva & $2(0.08)$ & & $2(0.38)$ & & \\
\hline \multirow[t]{5}{*}{ Urticaceae } & Cecropia concolor & Arbórea (plántula) & $4(0.17)$ & & & & $4(0.57)$ \\
\hline & Myriocarpa stipitata & Arbustiva & $5(0.21)$ & & & & $5(0.71)$ \\
\hline & Urera caracasana & Arbórea (plántula) & $13(0.55)$ & $6(1.12)$ & $5(0.96)$ & $2(0.33)$ & \\
\hline & Urera sp. & Arbustiva & $2(0.08)$ & $2(0.37)$ & & & \\
\hline & & & $2358(100)$ & $538(100)$ & $522(100)$ & $598(100)$ & 700 (100) \\
\hline
\end{tabular}

SAF simple = Sistema agroforestal simple-monocultivo; SAF intermedio = Sistema agroforestal intermedio; SAF complejo = Sistema agroforestal complejo; Bosque = Bosque secundario. 


\section{Apéndice 2}

Composición de la dieta de tres especies de roedores sigmodontinos en los diferentes sistemas de producción de cacao para la época de transición húmeda-seca. Se muestra la ocurrencia de individuos y frecuencia en porcentaje (en paréntesis) en cada sitio de muestreo.

\begin{tabular}{|c|c|c|c|c|c|c|c|c|c|c|c|c|}
\hline \multirow[t]{2}{*}{$\begin{array}{l}\text { Restos } \\
\text { Vegetales }\end{array}$} & \multirow[t]{2}{*}{ ITEMS } & \multirow[t]{2}{*}{ Abrev } & \multirow[t]{2}{*}{ Partes } & \multirow{2}{*}{$\begin{array}{l}\text { SAF } \\
\text { simple } \\
\text { Akodon da }\end{array}$} & \multirow{2}{*}{ Bosque } & $\begin{array}{l}\text { SAF } \\
\text { simple }\end{array}$ & \multirow[t]{2}{*}{$\begin{array}{l}\text { SAF } \\
\text { intermedio }\end{array}$} & \multirow[t]{2}{*}{$\begin{array}{l}\text { SAF } \\
\text { complejo }\end{array}$} & \multirow[t]{2}{*}{ Bosque } & \multirow{2}{*}{\multicolumn{2}{|c|}{\begin{tabular}{lr} 
SAF & \multicolumn{1}{c}{ SAF } \\
simple & complejo \\
& \\
Oligoryzomys & microtis
\end{tabular}}} & \multirow[t]{2}{*}{ Bosque } \\
\hline & & & & & & Euryoryzo & & & & & & \\
\hline \multicolumn{2}{|c|}{ Clase: Dicotyledoneae } & & & $n=5$ & $n=5$ & $n=6$ & $n=2$ & $n=3$ & $n=2$ & $n=31$ & $n=4$ & $n=6$ \\
\hline \multirow[t]{2}{*}{ Amaranthaceae } & Amaranthaceae sp.* & AMAt & Tallo & & & & & & & & $8(2.22)$ & \\
\hline & Iresine diffusa. & IDIt & Tallo & & & & $16(10.56)$ & $4(1.48)$ & & & & \\
\hline \multirow[t]{2}{*}{ Araliaceae } & Hydrocotyle sp. & HYDh & Hoja & $26(5.78)$ & & & & & & & & \\
\hline & & HYDt & Tallo & & & & & & & $132(4.73)$ & & \\
\hline Asclepiadaceae & Asclepiadaceae sp.* & ASCt & Tallo & & & & $14(7.78)$ & & $4(2.22)$ & $19(0.68)$ & $8(2.22)$ & \\
\hline \multirow[t]{2}{*}{ Convolvulaceae } & Ipomoea sp. & IPOt & Tallo & & & $7(1.30)$ & & & & $36(1.29)$ & & $63(11.67)$ \\
\hline & & IPOh & Hoja & & & & & & & $51(1.83)$ & & \\
\hline \multirow[t]{5}{*}{ Euphorbiaceae } & Acalypha arvensis & AARh & Hoja & & & & & & $16(8.89)$ & $69(2.47)$ & & \\
\hline & Acalypha stricta & ASTh & Hoja & $5(1.11)$ & $37(8.22)$ & & & & & & & \\
\hline & & ASTt & Tallo & & & & & & $22(12.30)$ & $86(3.08)$ & & \\
\hline & Acalyphasp. & ACAt & Tallo & & & $2(0.37)$ & & & & & & \\
\hline & & ACAh & Hoja & & & & & & & $74(2.65)$ & & \\
\hline \multirow[t]{2}{*}{ Fabaceae } & Kudan pueraria & & & & & & & & & & & \\
\hline & phaseoloides & KPPh & Hoja & & & $8(1.48)$ & & & & & & \\
\hline Plantaginaceae & Plantago sp. & PLAh & Hoja & & & $25(4.63)$ & & & & & & \\
\hline Papilionaceae & Desmodium sp. & DESh & Hoja & & & & & & $60(33.33)$ & & & \\
\hline Piperaceae & Piper aduncum & PADh & Hoja & & & & & & & & & $47(8.70)$ \\
\hline Polygonaceae & Polygonum sp. & POLt & Tallo & $10(2.22)$ & & & & & & & & $33(6.11)$ \\
\hline Rubiaceae & Borreria sp. & BORt & Tallo & & & & & & & $54(1.94)$ & & \\
\hline \multirow[t]{2}{*}{ Solanaceae } & Solanum sp. & SOLt & Tallo & & & & & & & & & $10(1.85)$ \\
\hline & & SOLh & Hoja & & & & & & & $72(2.58)$ & & \\
\hline Clase: Monocotyle & oneae & & & & & & & & & & & \\
\hline Alstroemeriaceae & Bomarea sp. & BOMh & Hoja & & & $6(1.11)$ & & & & $147(5.27)$ & & \\
\hline Araceae & Anthurium sp. & ANTh & Hoja & & $27(6.00)$ & & $16(8.89)$ & $12(4.44)$ & & $27(0.97)$ & $54(15.0)$ & \\
\hline Commelinaceae & Callisia repens & CREt & Tallo & & & & & & & $114(4.09)$ & & \\
\hline Costaceae & Costus scaber & $\mathrm{CSCh}$ & Hoja & & & & & & & $114(4.09)$ & & \\
\hline & & $\mathrm{CSCt}$ & Tallo & $25(5.56)$ & $7(1.56)$ & & & & & $11(0.39)$ & $35(9.72)$ & \\
\hline Cyperaceae & Cyperus chalaranthus & $\mathrm{CCHt}$ & Tallo & & & & $28(15.56)$ & $8(2.96)$ & & & & \\
\hline Poaceae & Axonopus compressus & ACOt & Tallo & & & & & & & $51(1.83)$ & $76(21.11)$ & \\
\hline & Imperata sp. & IMPt & Tallo & & & & & & & & $67(18.61)$ & \\
\hline & Oplismenus hirtellus & $\mathrm{OHIh}$ & Hoja & $14(3.11)$ & & & & & & & & \\
\hline & & OHIt & Tallo & & & & $7(3.89)$ & & 25 (13.89) & & $60(16.67)$ & \\
\hline & Paspalum & & & & & & & & & & & \\
\hline & conjugatum & PCOt & Tallo & & & & & & & $124(4.44)$ & & \\
\hline & & PCOh & Hoja & $8(1.78)$ & $28(6.22)$ & & & & & $23(0.82)$ & & \\
\hline & Paspalum & & & & & & & & & & & \\
\hline & paniculatum & PPAt & Tallo & $5(1.11)$ & $6(1.33)$ & & & & & $116(4.16)$ & & $108(20.0)$ \\
\hline & & PPAh & Hoja & & & & & & & $59(2.11)$ & & \\
\hline & Paspalum sp. & PASt & Tallo & & & $34(6.30)$ & $7(3.89)$ & & $6(3.36)$ & & & \\
\hline & Poaceae sp1. * & P01h & Hoja & & & $3(0.56)$ & & & & $87(3.12)$ & & \\
\hline & Poaceae sp2. * & $\mathrm{PO} 2 \mathrm{~h}$ & Hoja & & & $15(2.78)$ & & & & $65(2.33)$ & & \\
\hline & Sorghum & & & & & & & & & & & \\
\hline & arundinaceum & SARh & Hoja & $11(2.44)$ & & & & & & & & \\
\hline & & SARt & Tallo & & & & & & & & & $42(7.78)$ \\
\hline Semillas & & & & & & & & & & & & \\
\hline
\end{tabular}


Apéndice 2 continuación...

\begin{tabular}{|c|c|c|c|c|c|c|c|c|c|c|c|c|}
\hline \multirow[t]{2}{*}{$\begin{array}{l}\text { Restos } \\
\text { Vegetales }\end{array}$} & \multirow[t]{2}{*}{ ITEMS } & \multirow[t]{2}{*}{ Abrev } & \multirow[t]{2}{*}{ Partes } & $\begin{array}{c}\text { SAF } \\
\text { simple }\end{array}$ & Bosque & \multirow[t]{2}{*}{$\begin{array}{c}\text { SAF } \\
\text { simple }\end{array}$} & \multirow{2}{*}{\multicolumn{2}{|c|}{$\begin{array}{cc}\text { SAF } & \text { SAF } \\
\text { intermedio } & \text { complejo } \\
\text { Euryoryzomys nitidus }\end{array}$}} & \multirow[t]{2}{*}{ Bosque } & $\begin{array}{c}\text { SAF } \\
\text { simple }\end{array}$ & $\begin{array}{c}\text { SAF } \\
\text { complejo }\end{array}$ & Bosque \\
\hline & & & & \multicolumn{2}{|c|}{ Akodon dayi } & & & & & \multicolumn{3}{|c|}{ Oligoryzomys microtis } \\
\hline \multicolumn{13}{|c|}{ Clase: Dicotyledoneae } \\
\hline Polygonaceae & Polygonum sp. & POLs & Semilla & & & & $6(5.56)$ & $4(1.48)$ & & & & \\
\hline \multicolumn{13}{|l|}{ Espiguillas } \\
\hline \multicolumn{13}{|c|}{ Clase: Monocotyledoneae } \\
\hline \multirow[t]{2}{*}{ Cyperaceae } & Cyperus chalaranthus & $\mathrm{CCHI}$ & Lemma & & & $13(2.41)$ & & & & & & \\
\hline & & $\mathrm{CCHp}$ & Pálea & & & $7(1.30)$ & & & & & & \\
\hline \multirow[t]{12}{*}{ Poaceae } & Axonopus compressus & ACOp & Pálea & & & $8(1.48)$ & & & & & & \\
\hline & Paspalum & & & & & & & & & & & 123 \\
\hline & conjugatum & PCOes & Espiguilla & $22(4.89)$ & $15(3.33)$ & & & & & $6(0.22)$ & & (22.78) \\
\hline & & PCOg & Gluma & $4(0.89)$ & & & & & & & & \\
\hline & & PCOp & Pálea & $5(1.11)$ & & & & & & & & \\
\hline & Paspalum & & & & & & & & & & & \\
\hline & paniculatum & PPAes & Espiguilla & $23(5.11)$ & & & $22(12.20)$ & & & & & \\
\hline & & PPAg & Gluma & $17(3.78)$ & $2(0.44)$ & & & & & & & \\
\hline & & PPAp & Pálea & $12(2.67)$ & & & & & & & & \\
\hline & & PPAI & Lemma & $8(1.78)$ & & & & & & & & \\
\hline & & PPAa & Arista & $5(1.11)$ & & & & & & & & \\
\hline & Paspalum sp. & PASes & Espiguilla & & & $93(17.22)$ & & & & & & \\
\hline \multicolumn{13}{|l|}{ Micorrizas } \\
\hline \multicolumn{13}{|c|}{ Clase: Glomeromycetes } \\
\hline \multirow[t]{9}{*}{ Glomerales } & Glomus clavisporum & GCLe & Esporocarpo & & & & & $96(35.56)$ & & & & \\
\hline & Glomus fasciculatum & GFAe & Esporocarpo & & $46(10.22)$ & & & & & & & \\
\hline & & GFAhi & Hifas & & $25(5.56)$ & & & & & & & \\
\hline & Glomus macrocarpum & GMAe & Esporocarpo & & & & & & & $175(6.27)$ & & \\
\hline & & GMAhi & Hifas & & & & & & & $187(6.70)$ & & \\
\hline & Glomus sp1. & GL1e & Esporocarpo & & $32(7.11)$ & & & & & & & \\
\hline & & GL1hi & Hifas & & $21(4.67)$ & & & & & & & \\
\hline & Glomus sp2. & GL2e & Esporocarpo & $12(2.67)$ & & & & & & $147(5.27)$ & & \\
\hline & & GL2hi & Hifas & $20(4.44)$ & & & & & & $141(5.05)$ & & \\
\hline Artrópodos Ac & & & & & & & & & & & & \\
\hline Clase: Insecta & Coleóptera & $\mathrm{COL}$ & & $48(10.67)$ & $14(3.11)$ & $124(22.96)$ & & $58(21.48)$ & & & & \\
\hline & Díptera & DIP & & $28(6.23)$ & $28(6.22)$ & & & & & & & \\
\hline & Dyctioptera & DYC & & $20(4.44)$ & & & & & & & & \\
\hline & Formicidae & FOR & & & $55(12.22)$ & & & & & $33(1.18)$ & & \\
\hline & Hemiptera & HEM & & & & & & & & $27(0.97)$ & & \\
\hline & Hymenoptera & HYM & & $61(13.56)$ & $33(7.33)$ & $20(3.70)$ & & $37(13.70)$ & & $58(2.08)$ & & \\
\hline & Lepidoptera & LEP & & $13(2.89)$ & & & & & & & & \\
\hline Artrópodos lar & & & & & & & & & & & & \\
\hline & Hymenoptera larvas & HYL & & & & $39(7.22)$ & & & & & & \\
\hline Tejido Vascula & & & & & & & & & & & & \\
\hline & Morfotipo & MOR & & $22(4.89)$ & $37(8.23)$ & $6(1.11)$ & & $7(2.59)$ & & $79(2.59)$ & & \\
\hline Otros & Chlorophyta & $\mathrm{CHL}$ & Alga & & & & & & & & & $24(4.44)$ \\
\hline & Pelos & PEL & & $6(1.33)$ & $8(1.78)$ & $42(7.78)$ & $19(10.56)$ & $11(4.07)$ & $13(7.22)$ & $136(4.87)$ & $17(4.72)$ & $37(6.85)$ \\
\hline & Piedras & PIE & & $6(1.33)$ & $12(2.67)$ & $18(3.33)$ & $16(8.89)$ & $13(4.81)$ & $6(3.33)$ & $77(2.76)$ & $6(1.67)$ & $20(3.70)$ \\
\hline & Restos de avena & RAV & & $7(1.56)$ & $6(1.33)$ & $25(4.63)$ & $16(8.89)$ & $7(2.59)$ & $17(9.44)$ & $93(3.34)$ & $11(3.06)$ & $12(2.22)$ \\
\hline & Bolsa plástica & $\mathrm{BPL}$ & & & & & & & & $7(0.49)$ & $8(2.22)$ & \\
\hline & Indeterminado & IND & & $7(1.56)$ & $11(2.44)$ & $45(8.33)$ & $6(3.33)$ & $13(4.84)$ & $11(6.11)$ & $93(3.34)$ & $10(2.78)$ & 21 (3.89) \\
\hline
\end{tabular}




\section{Apéndice 3}

Índice del valor de importancia de recursos del orden/familias consumidos por los roedores Akodon dayi, Euryoryzomys nitidus y Oligoryzomys microtis. Se muestra el uso de recurso (Pxi) y el índice de valor de importancia de recursos (IVIR) en cada sitio de muestreo, se marcan con * los valores mayores a 0.1 y los resultados de IVIR mayores a 0.1 se encuentran resaltados con negrillas.

\begin{tabular}{|c|c|c|c|c|c|c|c|c|c|c|c|c|c|}
\hline & \multicolumn{4}{|c|}{ SAF simple } & \multicolumn{2}{|c|}{ SAF intermedio } & \multicolumn{2}{|c|}{ SAF complejo } & \multicolumn{3}{|c|}{ Bosque } & \multirow[b]{2}{*}{ A.da } & \multirow[b]{3}{*}{ IVIR } \\
\hline & O.mi & E.ni & A.da & & E.ni & & O.mi & E.ni & & O.mi & E.ni & & \\
\hline & & & & IVIR & & VIR & & & IVIR & & & & \\
\hline Orden/ Familia & Pxi & Pxi & Pxi & Pxi & & & Pxi & & Pxi & Pxi & Pxi & & \\
\hline Amaranthaceae & & & & & 0.092 & 0.092 & 0.022 & 0.015 & 0.037 & & & & \\
\hline Araliaceae & 0.047 & & 0.058 & 0.105 & & & & & & & & & \\
\hline Asclepiadaceae & 0.007 & & & 0.007 & 0.081 & 0.081 & 0.022 & & 0.022 & & 0.022 & & 0.022 \\
\hline Convolvulaceae & 0.031 & 0.013 & & 0.044 & & & & & & 0.117 & & & 0.117 \\
\hline Euphorbiaceae & 0.082 & 0.004 & 0.011 & 0.097 & & & & & & & $0.211^{*}$ & 0.074 & 0.285 \\
\hline Fabaceae & & 0.015 & & 0.015 & & & & & & & & & \\
\hline Plantaginaceae & & 0.046 & & 0.046 & & & & & & & & & \\
\hline Papilionaceae & & & & & & & & & & & $0.333^{*}$ & & 0.333 \\
\hline Piperaceae & & & & & & & & & & 0.087 & & & 0.087 \\
\hline Polygonaceae & & & 0.022 & 0.022 & 0.035 & 0.035 & & 0.015 & 0.015 & 0.061 & & 0.105 & 0.166 \\
\hline Rubiaceae & 0.019 & & & 0.019 & & & & & & & & & \\
\hline Solanaceae & 0.026 & & & 0.026 & & & & & & 0.019 & & & 0.019 \\
\hline Alstroemeriaceae & 0.053 & 0.011 & & 0.064 & & & & & & & & & \\
\hline Araceae & 0.010 & & & 0.010 & 0.092 & 0.092 & 0.150 & 0.044 & 0.194 & & & 0.054 & 0.054 \\
\hline Commelinaceae & 0.041 & & & 0.041 & & & & & & & & & \\
\hline Costaceae & 0.045 & & 0.056 & 0.100 & & & 0.097 & & 0.097 & & & 0.014 & 0.014 \\
\hline Cyperaceae & & 0.037 & & 0.037 & 0.162 & 0.162 & & 0.030 & 0.030 & & & & \\
\hline Poaceae & 0.190 & $0.283^{*}$ & $0.298^{*}$ & 0.771 & $0.208^{*}$ & 0.208 & $0.564^{*}$ & & 0.564 & $0.506^{*}$ & 0.172 & 0.101 & 0.779 \\
\hline Glomerales & $0.233^{*}$ & & 0.071 & 0.304 & & & & $0.356^{*}$ & 0.356 & & & $0.247^{*}$ & 0.247 \\
\hline Coleóptera & & $0.230^{*}$ & 0.107 & 0.336 & & & & $0.215^{*}$ & 0.215 & & & 0.028 & 0.028 \\
\hline Díptera & & & 0.062 & 0.062 & & & & & & & & 0.056 & 0.056 \\
\hline Dyctioptera & & & 0.044 & 0.044 & & & & & & & & & \\
\hline Formicidae & 0.012 & & & 0.012 & & & & & & & & 0.109 & 0.109 \\
\hline Hemiptera & 0.010 & & & 0.010 & & & & & & & & & \\
\hline Hymenoptera & 0.021 & 0.037 & 0.136 & 0.193 & & & & 0.137 & 0.137 & & & 0.066 & 0.066 \\
\hline Lepidoptera & & & 0.029 & 0.029 & & & & & & & & & \\
\hline $\begin{array}{l}\text { Hymenoptera } \\
\text { pupas }\end{array}$ & & 0.072 & & 0.072 & & & & & & & & & \\
\hline Tejido Vascular & 0.028 & 0.011 & 0.049 & 0.088 & & & & 0.026 & 0.026 & & & 0.074 & 0.074 \\
\hline Otros & 0.146 & $0.241^{*}$ & 0.058 & 0.444 & $0.329^{*}$ & 0.329 & 0.144 & 0.163 & 0.307 & $0.211^{*}$ & $0.261^{*}$ & 0.074 & 0.546 \\
\hline
\end{tabular}

SAF simple = Sistema agroforestal simple monocultivo; SAF intermedio = Sistema agroforestal intermedio; SAF complejo = Sistema agroforestal complejo; Bosque $=$ Bosque secundario. O.mi $=$ Oligoryzomys microtis; E.ni $=$ Euryoryzomys nitidus; $\mathrm{A} . \mathrm{da}=$ Akodon dayi . 